\title{
Pentamethyldisilanyl-substituierte Cyclopentadiene: Synthese, Struktur und dynamisches Verhalten*
}

\author{
P. Jutzi, J. Kleimeier, R. Krallmann, H.-G. Stammler und B. Neumann \\ Fakultät für Chemie der Universität Bielefeld, Universitätsstr., D-33615 Bielefeld (Deutschland) \\ (Eingegangen den 15. März 1993)
}

\begin{abstract}
The pentamethyldisilanyl-substituted cyclopentadienes $\mathrm{Me}_{n} \mathrm{C}_{5} \mathrm{H}_{6-n-m}\left(\mathrm{Si}_{2} \mathrm{Me}_{5}\right)_{m}$ (for $n=0: 1(m=1), 2(m=2), 3(m=3), 4$ $(m=4)$; for $n=1: 5(m=1), 7(m=2), 9(m=3)$; for $n=3: 13(m=1), 14(m=2)$; for $n=4: 15(m=1))$ are accessible in good yields by treatment of the corresponding cyclopentadienyllithium compounds with $\mathrm{Me}_{5} \mathrm{Si}_{2} \mathrm{Cl}$. The mono- $\mathrm{Me}_{5} \mathrm{Si}_{2}$-substituted species $\mathbf{1}$ and $\mathbf{5}$ are present only to a small extend in form of vinylic isomers and to a greater extend as isomers with the $\mathrm{Me}_{5} \mathrm{Si}_{2}-\mathrm{group}$ in allylic position; the latter possess a dynamic structure due to sigmatropic rearrangements. In the twice-Me ${ }_{5} \mathrm{Si}_{2}$-substituted cyclopentadienes 2 and 7, the 5,5 and 2,5 isomers are observed, which can be interconverted by silatropic shifts; in addition, the presence of two vinylic isomers can be proved in the case of 2 . In the cyclopentadiene species 3 and 9 with three $\mathrm{Me}_{5} \mathrm{Si}_{2}$ groups, only the 2,5,5 isomers can be detected by NMR spectroscopy. Compound 3 possesses a fluxional structure and can thus be deprotonated. On the other hand, 9 does not show a fluxional behaviour and thus cannot be deprotonated. The cyclopentadiene 4 with four $\mathrm{Me}_{5} \mathrm{Si}_{2}$ substituents possesses a static structure and cannot be deprotonated. The 2,3,5,5 position of the substituents is proved by an X-ray crystal structure analysis. Only two $\mathrm{Me}_{5} \mathrm{Si}_{2}$ groups can be incorporated in the carbon skeleton of 1,2,4-trimethylcyclopentadiene, whereby compounds of the type 1,2,4- $\mathrm{Me}_{3} \mathrm{C}_{5} \mathrm{H}_{3-n}\left(\mathrm{Si}_{2} \mathrm{Me}_{5}\right)_{n}(13: n=1 ; 14: n=2)$ are formed. Surprisingly, 14 cannot be deprotonated with ${ }^{\mathrm{n}} \mathrm{BuLi}$ and $\mathrm{KH}$, respectively. The reaction of $\mathrm{Me}_{4} \mathrm{C}_{5} \mathrm{HLi}$ with $\mathrm{Me}_{5} \mathrm{Si}_{2} \mathrm{Cl}$ leads to the cyclopentadiene $\mathrm{Me}_{4} \mathrm{C}_{5} \mathrm{HSi}_{2} \mathrm{Me}_{5}$ (15). Though compound 15 can be deprotonated, further reaction of the resulting anion with $\mathrm{Me}_{5} \mathrm{Si}_{2} \mathrm{Cl}$ does not lead to the expected cyclopentadiene $\mathrm{Me}_{4} \mathrm{C}_{5}\left(\mathrm{Si}_{2} \mathrm{Me}_{5}\right)_{2}$ (16). On the other hand, 16 can be prepared by metallation of 14 with $\mathrm{C}_{8} \mathrm{~K}$ and further reaction with $\mathrm{CH}_{3} \mathrm{I}$. In contrast to 14, compound 4 cannot be deprotonated with $\mathrm{C}_{8} \mathrm{~K}$; the reaction of 4 with $\mathrm{C}_{8} \mathrm{~K}$ and $\mathrm{CH}_{3} \mathrm{I}$ leads to 9 via $\mathrm{Si}-\mathrm{C}$ bond splitting. The pentamethyldisilanyl-substituted pentamethylcyclopentadiene $\mathrm{Me}_{5} \mathrm{C}_{5} \mathrm{Si}_{2} \mathrm{Me}_{5}$ (17) is obtained by reaction of $\mathrm{Me}_{5} \mathrm{C}_{5} \mathrm{~K}$ with $\mathrm{Me}_{5} \mathrm{Si}_{2} \mathrm{Cl}$; compound 17 shows dynamic behaviour; the migration of the $\mathrm{Me}_{5} \mathrm{Si}_{2}$ group is slower than that of the $\mathrm{Me}_{3} \mathrm{Si}$ group in $\mathrm{Me}_{5} \mathrm{C}_{5} \mathrm{SiMe}_{3}$. Three ElMe $\mathrm{Me}_{3}$ groups can be introduced stcpwisc into the 1,2,4-Me $\mathrm{M}_{5} \mathrm{H}_{3}$ molecule, as demonstrated by the exemplary synthesis of the cyclopentadienes 1,2,4$\mathrm{Me}_{3} \mathrm{C}_{5} \mathrm{H}_{3-n}\left(\mathrm{SiMe}_{3}\right)_{n}(10: n=1 ; 11: n=2)$ and $1,2,4-\mathrm{Me}_{3} \mathrm{C}_{5}\left(\mathrm{SiMe}_{3}\right)_{2} \mathrm{SnMe}_{3}(12)$.
\end{abstract}

\section{Zusammenfassung}

Die $\mathrm{Si}_{2} \mathrm{Me}_{5}$-substituierten Cyclopentadiene $\mathrm{Me}_{n} \mathrm{C}_{5} \mathrm{H}_{6-n-m}\left(\mathrm{Si}_{2} \mathrm{Me}_{5}\right)_{m}$ (für $n=0: 1(m=1), 2(m=2), 3(m=3), 4(m=4)$; für $n=1: 5(m=1), 7(m=2), 9(m=3)$; für $n=3: 13(m=1), 14(m=2)$; für $n=4: 15(m=1))$ sind durch Umsetzung der entsprechenden Cyclopentadienyl-Lithium-Verbindung mit $\mathrm{Me}_{5} \mathrm{Si}_{2} \mathrm{Cl}$ in guten Ausbeuten zugänglich. In den einfach $\mathrm{Si}_{2} \mathrm{Me}_{5}$-substituierten Systemen 1 und 5 findet man zu einem geringen Anteil Isomere mit vinylständiger $\mathrm{Si}_{2} \mathrm{Me}_{5}$-Gruppe und zu einem überwiegenden Anteil das Isomer mit allylständiger $\mathrm{Si}_{2} \mathrm{Me}_{5}$-Gruppe, welches aufgrund von sigmatropen Umlagerungen eine dynamische Struktur besitzt. In den zweifach $\mathrm{Si}_{2} \mathrm{Me}_{5}$-substituierten Cyclopentadienen 2 und 7 beobachtet man die jeweiligen 5,5und 2,5-Isomere, welche durch Silatropie miteinander im Gleichgewicht stehen; zusätzlich lassen sich in 2 noch zwei Isomere mit ausschließlich vinylständigen Substituenten nachweisen. In den dreifach $\mathrm{Si}_{2} \mathbf{M e}_{5}$-substituierten Systemen 3 und 9 ist nur das 2,5,5-Isomere nachweisbar. 3 besitzt eine dynamische Struktur und ist deshalb deprotonierbar. 9 hingegen ist nicht dynamisch und aufgrund des Fehlens einer Allyl-H-Funktion nicht deprotonierbar. Auch das vierfach $\mathrm{Si}_{2} \mathrm{Me}_{5}$-substituierte Cyclopentadien 4 zeigt keine Moleküldynamik und kann nicht deprotoniert werden; die 2,3,5,5-Anordnung der Substituenten in 4 wird anhand einer Röntgenstrukturanalyse belegt. Im 1,2,4-Trimethylcyclopentadien gelingt jedoch nur die Einführung von $\mathrm{zwei}^{\mathrm{Si}} \mathrm{Me}_{5}$-Gruppen,

Correspondence to: Dr. P. Jutzi.

* Herrn Professor M.F. Lappert zum 65. Geburtstag gewidmet. 
wobei die Verbindungen des Typs 1,2,4- $\mathrm{Me}_{3} \mathrm{C}_{5} \mathrm{H}_{3-n}\left(\mathrm{Si}_{2} \mathrm{Me}_{5}\right)_{n}(13: n=1 ; 14: n=2)$ entstehen. Überraschenderweise ist 14 mit ${ }^{n} \mathrm{BuLi}$ oder $\mathrm{KH}$ nicht deprotonierbar. Die Umsetzung von $\mathrm{Me}_{4} \mathrm{C}_{5} \mathrm{HLi}$ mit $\mathrm{Me}_{5} \mathrm{Si}_{2} \mathrm{Cl}$ führt zum Cyclopentadien $\mathrm{Me}_{4} \mathrm{C}_{5} \mathrm{HSi}_{2} \mathrm{Me}_{5}$ (15). Obwohl 15 deprotonierbar ist, gelingt durch Umsetzung des Anions mit $\mathrm{Me}_{5} \mathrm{Si}_{2} \mathrm{Cl}$ die Synthese von $\mathrm{Me}_{4} \mathrm{C}_{5}\left(\mathrm{Si}_{2} \mathrm{Me}_{5}\right)_{2}(16)$ nicht. Verbindung 16 läßt sich allerdings durch Metallierung von 14 mit $\mathrm{C}_{8} \mathrm{~K}$ und anschließende Umsetzung mit $\mathrm{CH}_{3} \mathrm{I}$ darstellen. Im Gegensatz dazu kann 4 mit $\mathrm{C}_{8} \mathrm{~K}$ nicht deprotoniert werden; die Umsetzung mit $\mathrm{C}_{8} \mathrm{~K}$ und $\mathrm{CH}_{3} \mathrm{I}$ läuft über Si-C-Bindungsspaltung zu 9. Das Cyclopentadienyldisilan $\mathrm{Me}_{5} \mathrm{C}_{5} \mathrm{Si}_{2} \mathrm{Me}_{5}$ (17) erhält man durch Umsetzung von $\mathrm{Me}_{5} \mathrm{C}_{5} \mathrm{~K}$ mit $\mathrm{Me}_{5} \mathrm{Si}_{2} \mathrm{Cl}_{3} 17$ zeigt dynamisches Verhalten, die Wanderungsgeschwindigkeit der $\mathrm{Si}_{2} \mathrm{Me}_{5}$-Gruppe ist geringer als die der $\mathrm{SiMe}_{3}$-Gruppe im Cyclopentadienylsilan $\mathrm{Me}_{5} \mathrm{C}_{5} \mathrm{SiMe}_{3}$. Im Cyclopentadien 1,2,4-Me $\mathrm{C}_{5} \mathrm{H}_{3}$ lassen sich sukzessiv drei $\mathrm{ElMe}_{3}$-Gruppen $(\mathrm{El}=\mathrm{Si}, \mathrm{Sn})$ einführen, wie durch die beispielhalfte Synthese von 1,2,4-Me ${ }_{3} \mathrm{C}_{5} \mathrm{H}_{3-n}\left(\mathrm{SiMe}_{3}\right)_{n}(10: n=1,11: n=2)$ und 1,2,4-Me ${ }_{3} \mathrm{C}_{5}\left(\mathrm{SiMe}_{3}\right)_{2} \mathrm{SnMe}_{3}(12)$ gezeigt wird.

\section{Einleitung}

Trimethylsilyl-substituierte Cyclopentadiene sind seit langem bekannt und haben in vielfältiger Form Eingang in die Komplexchemie von s-, p-, d- und f-Elementen gefunden [1]. Wichtige Untersuchungen auf diesem Gebiet stammen aus der Arbeitsgruppe von Lappert [1a]. Die hervorstechendsten Eigenschaften entsprechender Cyclopentadienyl-Komplexe sind gute Löslichkeit auch in unpolaren Solventien und gute Kristallisationseigenschaften. Die $\mathrm{SiMe}_{3}$-Gruppe zeigt sowohl Elektronendonor- als auch Elektronenakzeptor-Verhalten. Die Akzeptor-Eigenschaft macht sich in der NMR-Spektroskopie durch Entschirmung der ${ }^{13} \mathrm{C}$ Kerne des $\mathrm{SiMe}_{3}$-substituierten Cyclopentadienylsystems und damit durch Tieffeldverschiebung der Resonanzen bemerkbar. Die Donorfunktion spiegelt sich in den Energien von Metall-d-Elektronen entsprechender Metallocene wider [2]; mit zunehmendem Silylierungsgrad der Liganden erhöht sich die Elektronendichte am Metallzentrum.

Der Einbau von Pentamethyldisilanyl-Gruppen anstelle der Trimethylsilyl-Gruppen sollte $\mathrm{zu}$ interessanten Eigenschaftsänderungen in entsprechenden Metallkomplexen führen. So könnte die Löslichkeit in unpolaren Solventien noch verstärkt werden. Darüber hinaus ist bei gecigneter Konformation der DisilanylEinheiten eine kinetische Stabilisierung durch gute Abschirmung des Komplex-Zentrums denkbar. Schließlich sind durch den Einbau von Si-Si-Einheiten interessante elektronische Effekte zu erwarten.

Das bisher einzige literaturbekannte Cyclopentadien mit einer Pentamethyldisilanyl-Gruppe ist zugleich der Grundkörper dieser Verbindungsklasse, das Pentamethyldisilanylcyclopentadien (1) [3]. Diese Verbindung wurde bereits 1964 durch Umsetzung von Cyclopentadienyl-Magnesiumbromid mit Chlorpentamethyldisilan synthetisiert. Spektroskopische Daten sind jedoch bisher nicht veröffentlicht worden. Mit deprotoniertem 1 als Komplexligand sind nur wenige Verbindungen bekannt [3].

Ausgehend von Cyclopentadien sowie von methylsubstituierten Cyclopentadienen haben wir neue Pen- tamethyldisilanylcyclopentadiene dargestellt, über deren Synthese, Struktur und Dynamik wir im folgenden berichten.

\section{Ergebnisse}

Als generelle Synthesemethode wählen wir die Lithiierung des entsprechenden Cyclopentadiens mit n-Butyllithium in THF und anschließende Umsetzung mit Chlorpentamethyldisilan. Diese Methode erweist sich auch, von zwei Ausnahmen abgesehen, als erfolgreich.

Durch Umsetzung von Cyclopentadienyl-Lithium mit Chlorpentamethyldisilan erhält man Verbindung 1 in 61\%iger Ausbeute als kurzfristig an der Luft handhabbare farblose Flüssigkeit (Gl. (1)). NMR-spektroskopisch findet man analog zum Trimethylsilylcyclopentadien drei Isomere. Aus den Integralverhältnissen lassen sich die ungefähren Anteile der Isomeren bestimmen. Das Hauptisomer 1a mit der Disilanylgruppe in AllylStellung ist mit einem Anteil von $80 \%$ vertreten. Die Isomere $1 \mathbf{b}$ und 1c mit der Disilanylgruppe in VinylStellung liegen zu $15 \%$ bzw. zu $5 \%$ vor. Im Vergleich zum Trimethylsilylcyclopentadien ist die IsomerenVerteilung zugunsten der Vinylisomeren verschoben [4]. Das Isomer 1a zeigt sich in Lösung hochdynamisch, bei den Vinylisomeren läßt sich im betrachteten Temperaturintervall $\left(-80\right.$ bis $\left.+100^{\circ} \mathrm{C}\right)$ keine Dynamik beobachten. Verbindung 1 läßt sich wegen der in allen Isomeren vorhandenen Allyl-Wasserstoffatome sehr leicht mit n-Butyllithium deprotonieren. Die so entstandene Lithium-Verbindung ist in unpolaren Solventien schwer und in THF sehr gut löslich.

Setzt man die Lithium-Verbindung von 1 mit Chlorpentamethyldisilan in THF um, so erhält man das zweifach disilanyl-substituierte 2 (Gl. (2)) in 76\%iger Ausbeute 2 als klare ölige Flüssigkeit, die kurzfristig an der Luft handhabbar ist. Die NMR-Daten belegen das Vorliegen des 5,5-Isomeren (2a) sowie des 2,5-Isomeren (2b) im Verhältnis 3/1. Die Isomere 2a und $\mathbf{2 b}$ stehen durch Silatropie miteinander im Gleichgewicht. Ferner lassen sich in geringen Mengen zwei Isomere mit jeweils zwei vinylisch gebundenen Pentamethyldisi- 
lanyl-Gruppen nachweisen. Im analogen Bis(trimethylsilyl)cyclopentadien ist der Anteil der Vinylisomeren bei Raumtemperatur wesentlich geringer (ca. 1/40) [5]. Aufgrund des Gleichgewichtes zwischen 2a und 2b ist Verbindung 2 vollständig deprotonierbar. Die Lithium-Verbindung von 2 ist in unpolaren Solventien schwer und in THF sehr gut löslich.

Die Umsetzung der Lithium-Verbindung von 2 mit Chlorpentamethyldisilan liefert das dreifach disilanylsubstituierte Cyclopentadien 3 in $74 \%$ iger Ausbeute als schwach braun gefärbtes Öl, das kurzzeitig an der Luft handhabbar ist (Gl. (3)). Die NMR-Daten sprechen eindeutig für das Vorliegen des 2,5,5-Isomeren. Weitere Isomere lassen sich spektroskopisch nicht nachweisen. Die vorhandene Moleküldynamik zeigt sich anhand der erst bei $100^{\circ} \mathrm{C}$ beobachtbaren chemischen Äquivalenz aller drei Pentamethyldisilanyl-Gruppen. Damit verhält sich 3 analog dem vergleichbaren Tris(trimethylsilyl)cyclopentadien [6]. Im Raumtemperatur- ${ }^{1} \mathrm{H}-\mathrm{NMR}$-Spektrum von 3 beobachtet man für die Protonen der allylischen $\mathrm{SiMe}_{2}$-Gruppen zwei Singuletts bei 0.14 und $0.15 \mathrm{ppm}$. Diese Aufspaltung ist wohl auf unterschiedliche Wechselwirkungen mit der vinylischen Disilanylgruppe zurückzuführen (Diastereotopie). Obwohl NMRspektroskopisch kein Isomer mit allylständigem Wasserstoffatom nachweisbar ist, läßt sich Verbindung 3 deprotonieren. Bisher ist es trotz Variation von Lösungsmittel und Deprotonierungsagens nicht gelungen, Verbindung 3 vollständig zu deprotonieren. Das beste Ergebnis wurde mit einem 1.5-fachen Überschuß an $\mathrm{n}$-Butyllithium in THF erzielt $(80 \%$ Deprotonierung). Die Lithium-Verbindung ist auch in unpolaren Solventien löslich.

Setzt man die Lithium-Verbindung von 3 mit Chlorpentamethyldisilan um, so erhält man nach gängiger Aufarbeitung und Kristallisation das vierfach disilanylsubstituierte Cyclopentadien 4 als farblose, luftstabile Kristalle in 50\%iger Ausbeute (Gl. (3)). NMR-spektroskopisch läßt sich nur das Vorliegen des 2,3,5,5-Iso-

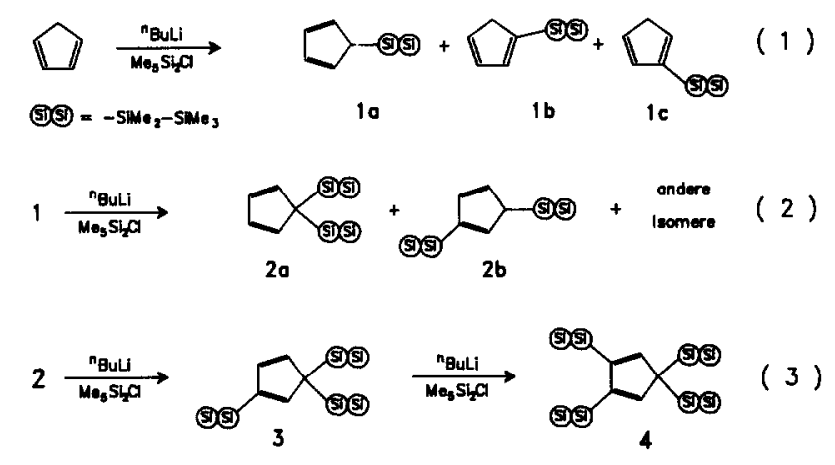

Schema 1.

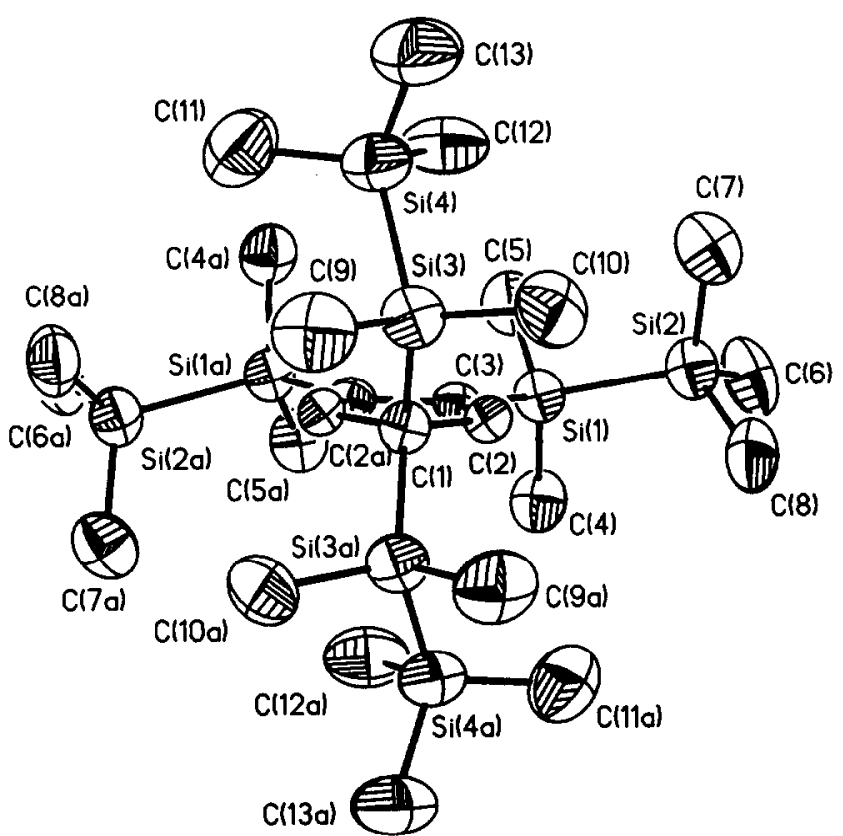

Abb. 1. Kristallstruktur von 4.

mers nachweisen. Im Temperaturbereich von -80 bis $+100^{\circ} \mathrm{C}$ findet man keine Hinweise für ein dynamisches Verhalten. Mit den Deprotonierungsagentien nButyllithium, Kaliumhydrid, Natrium und dem Kaliumgraphit $\mathrm{C}_{8} \mathrm{~K}$ läßt sich das Cyclopentadien 4 entsprechend dem Tetrakis(trimethylsilyl)cyclopentadien nicht deprotonieren [7].

Zur Röntgenstrukturanalyse geeignete Kristalle der Verbindung 4 können durch Kristallisieren aus Dichlormethan/Acetonitril erhalten werden $\left[8^{*}\right]$. Abbildung 1 zeigt die Molekülstruktur von 4 . Die wichtigsten Daten zur Kristallstruktur von 4 sind in den Tabellen 1-5 zusammengestellt. Verbindung 4 kristallisiert in der tetragonalen Raumgruppe $I 4_{1} / a$. Die Elementarzelle enthält 8 Moleküle der erwarteten Zusammensetzung. C-C-Einfach- und -Doppelbindungen des Cyclopentadienyl-Gerüsts sind deutlich voneinander unterscheidbar. Die gefundenen C-C-Abstände sind charakteristisch für Cyclopentadien-Systeme. Die $\mathrm{Si}$ Si-Abstände in den vier Disilanyl-Einheiten liegen mit $2.34 \AA$ im erwarteten Bereich, ebenso die $\mathrm{Si}-\mathrm{C}$ Abstände zwischen $\mathrm{Si}(1)$ und $\mathrm{C}(3)$ bzw. zwischen $\mathrm{Si}(1 \mathrm{a})$ und $C(3 a)$ mit $1.88 \AA$. Dagegen ist die Si-C-Bindung sowohl zwischen $\mathrm{Si}(3)$ und $\mathrm{C}(1)$ als auch zwischen Si(3a) und C(1) mit $1.92 \AA$ etwas verlängert. Diese Verlängerung ist wohl auf die Anwesenheit einer zweiten sterisch anspruchsvollen pentamethyldisilanylGruppe am Allyl-Kohlenstoffatom zurückzuführen.

\footnotetext{
* Die Literaturnummer mit einem Sternchen deutet eine Bemerkung in der Literaturliste an.
} 
TABELLE 1. Atomkoordinaten $\left(\times 10^{4}\right)$ und äquivalente isotrope Auslenkunsparameter $\left(\AA^{2} \times 10^{3}\right)$ in 4

\begin{tabular}{lrlrr}
\hline Atom & \multicolumn{1}{l}{$x$} & \multicolumn{1}{l}{$z$} & \multicolumn{1}{l}{$U_{\text {eq }}{ }^{\mathrm{a}}$} \\
\hline $\mathrm{Si}(1)$ & $9153(1)$ & $6908(1)$ & $147(1)$ & $53(1)$ \\
$\mathrm{Si}(2)$ & $8446(1)$ & $5985(1)$ & $464(1)$ & $64(1)$ \\
$\mathrm{Si}(3)$ & $10493(1)$ & $6813(1)$ & $2308(1)$ & $60(1)$ \\
$\mathrm{Si}(4)$ & $11308(1)$ & $6205(1)$ & $1715(1)$ & $75(1)$ \\
$\mathrm{C}(1)$ & 10000 & 7500 & $1837(4)$ & $39(4)$ \\
$\mathrm{C}(2)$ & $9527(3)$ & $7145(3)$ & $1402(3)$ & $39(3)$ \\
$\mathrm{C}(3)$ & $9693(3)$ & $7270(3)$ & $798(3)$ & $40(3)$ \\
$\mathrm{C}(4)$ & $8572(3)$ & $7589(3)$ & $-189(3)$ & $73(3)$ \\
$\mathrm{C}(5)$ & $9703(3)$ & $6539(3)$ & $-497(3)$ & $77(4)$ \\
$\mathrm{C}(6)$ & $7911(4)$ & $5780(4)$ & $-246(3)$ & $99(4)$ \\
$\mathrm{C}(7)$ & $8981(4)$ & $5211(3)$ & $667(3)$ & $86(4)$ \\
$\mathrm{C}(8)$ & $7828(3)$ & $6167(4)$ & $1109(3)$ & $89(4)$ \\
$\mathrm{C}(9)$ & $10981(3)$ & $7220(4)$ & $2960(3)$ & $91(4)$ \\
$\mathrm{C}(10)$ & $9864(4)$ & $6167(3)$ & $2639(3)$ & $88(4)$ \\
$\mathrm{C}(11)$ & $12090(4)$ & $6739(4)$ & $1562(3)$ & $112(5)$ \\
$\mathrm{C}(12)$ & $10978(4)$ & $5853(4)$ & $974(3)$ & $110(4)$ \\
$\mathrm{C}(13)$ & $11575(4)$ & $5448(4)$ & $2204(4)$ & $127(5)$ \\
\hline
\end{tabular}

${ }^{a}$ Äquivalente isotrope $U$ berechnet als ein Drittel der Spur des orthogonalen $U_{i j}$-Tensors.

TABELLE 2. Bindungslängen $(\AA)$ in 4

\begin{tabular}{llll}
\hline $\mathrm{Si}(1)-\mathrm{Si}(2)$ & $2.341(3)$ & $\mathrm{Si}(1)-\mathrm{C}(3)$ & $1.879(6)$ \\
$\mathrm{Si}(1)-\mathrm{C}(4)$ & $1.870(7)$ & $\mathrm{Si}(1)-\mathrm{C}(5)$ & $1.885(7)$ \\
$\mathrm{Si}(2)-\mathrm{C}(6)$ & $1.886(7)$ & $\mathrm{Si}(2)-\mathrm{C}(7)$ & $1.864(7)$ \\
$\mathrm{Si}(2)-\mathrm{C}(8)$ & $1.864(7)$ & $\mathrm{Si}(3)-\mathrm{Si}(4)$ & $2.340(4)$ \\
$\mathrm{Si}(3)-\mathrm{C}(1)$ & $1.920(5)$ & $\mathrm{Si}(3)-\mathrm{C}(9)$ & $1.863(7)$ \\
$\mathrm{Si}(3)-\mathrm{C}(10)$ & $1.878(7)$ & $\mathrm{Si}(4)-\mathrm{C}(11)$ & $1.854(8)$ \\
$\mathrm{Si}(4)-\mathrm{C}(12)$ & $1.847(8)$ & $\mathrm{Si}(4)-\mathrm{C}(13)$ & $1.870(8)$ \\
$\mathrm{C}(1)-\mathrm{C}(2)$ & $1.475(8)$ & $\mathrm{C}(1)-\mathrm{Si}(3 \mathrm{~A})$ & $1.920(5)$ \\
$\mathrm{C}(1)-\mathrm{C}(2 \mathrm{~A})$ & $1.475(8)$ & $\mathrm{C}(2)-\mathrm{C}(3)$ & $1.361(8)$ \\
$\mathrm{C}(3)-\mathrm{C}(3 \mathrm{~A})$ & $1.479(12)$ & & \\
\hline
\end{tabular}

Methylcyclopentadien liegt als Gemisch aus drei deprotonierbaren Isomeren vor. Setzt man die Lithium-Verbindung des Methylcyclopentadiens mit

TABELLE 3. Bindungswinkel $\left(^{\circ}\right)$ in 4

\begin{tabular}{|c|c|c|c|}
\hline $\mathrm{Si}(2)-\mathrm{Si}(1)-\mathrm{C}(3)$ & $112.7(2)$ & $\mathrm{Si}(2)-\mathrm{Si}(1)-\mathrm{C}(4)$ & $107.3(2)$ \\
\hline$C(3)-S i(1)-C(4)$ & 111.1(3) & $\mathrm{Si}(2)-\mathrm{Si}(1)-\mathrm{C}(5)$ & $104.7(2)$ \\
\hline$C(3)-S i(1)-C(5)$ & $112.2(3)$ & $C(4)-S i(1)-C(5)$ & $108.5(3)$ \\
\hline $\mathrm{Si}(1)-\mathrm{Si}(2)-\mathrm{C}(6)$ & $103.9(2)$ & $\mathrm{Si}(1)-\mathrm{Si}(2)-\mathrm{C}(7)$ & $110.8(2)$ \\
\hline$C(6)-S i(2)-C(7)$ & $108.9(3)$ & Si(1)-Si(2)-C(8) & $116.5(2)$ \\
\hline$C(6)-S i(2)-C(8)$ & $107.1(3)$ & $C(7)-S i(2)-C(8)$ & $109.2(3)$ \\
\hline $\mathrm{Si}(4)-\mathrm{Si}(3)-\mathrm{C}(1)$ & $112.9(2)$ & $\mathrm{Si}(4)-\mathrm{Si}(3)-\mathrm{C}(9)$ & $106.6(2)$ \\
\hline$C(1)-S i(3)-C(9)$ & $110.9(3)$ & $\mathrm{Si}(4)-\mathrm{Si}(3)-C(10)$ & $108.0(2)$ \\
\hline $\mathrm{C}(1)-\mathrm{Si}(3)-\mathrm{C}(10)$ & $109.8(2)$ & $C(9)-S i(3)-C(10)$ & $108.6(3)$ \\
\hline $\mathrm{Si}(3)-\mathrm{Si}(4)-C(11)$ & $111.4(3)$ & $\mathrm{Si}(3)-\mathrm{Si}(4)-\mathrm{C}(12)$ & $115.1(3)$ \\
\hline $\mathrm{C}(11)-\mathrm{Si}(4)-\mathrm{C}(12)$ & $109.3(4)$ & $\mathrm{Si}(3)-\mathrm{Si}(4)-C(13)$ & $105.4(3)$ \\
\hline $\mathrm{C}(11)-\mathrm{Si}(4)-\mathrm{C}(13)$ & $108.0(4)$ & $C(12)-S i(4)-C(13)$ & $107.2(4)$ \\
\hline$S i(3)-C(1)-C(2)$ & $108.7(2)$ & $\mathrm{Si}(3)-C(1)-\mathrm{Si}(3 \mathrm{~A})$ & $116.2(4)$ \\
\hline $\mathrm{C}(2)-\mathrm{C}(1)-\mathrm{Si}(3 \mathrm{~A})$ & $110.5(2)$ & $\mathrm{Si}(3)-C(1)-C(2 \mathrm{~A})$ & $110.5(2)$ \\
\hline$C(2)-C(1)-C(2 A)$ & 101.1(7) & $\mathrm{Si}(3 \mathrm{~A})-\mathrm{C}(1)-C(2 \mathrm{~A})$ & $108.7(2)$ \\
\hline$C(1)-C(2)-C(3)$ & $112.4(5)$ & $S i(1)-C(3)-C(2)$ & $121.2(5)$ \\
\hline$S i(1)-C(3)-C(3 A)$ & $131.7(2)$ & $C(2)-C(3)-C(3 A)$ & $107.0(4)$ \\
\hline
\end{tabular}

TABELLE 4. Anisotrope Auslenkungsparameter $\left(\AA^{2} \times 10^{3}\right)$ in $4^{\text {a }}$

\begin{tabular}{lrrrrrr}
\hline Atom & \multicolumn{1}{c}{$U_{11}$} & \multicolumn{1}{c}{$U_{22}$} & \multicolumn{1}{c}{$U_{33}$} & \multicolumn{1}{c}{$U_{12}$} & \multicolumn{1}{c}{$U_{13}$} & \multicolumn{1}{c}{$U_{23}$} \\
\hline Si(1) & $61(2)$ & $59(2)$ & $40(1)$ & $-4(1)$ & $-6(1)$ & $1(1)$ \\
Si(2) & $70(2)$ & $68(2)$ & $55(1)$ & $-12(1)$ & $-12(1)$ & $6(1)$ \\
Si(3) & $68(2)$ & $68(2)$ & $43(1)$ & $1(1)$ & $-8(1)$ & $6(1)$ \\
Si(4) & $72(2)$ & $76(2)$ & $77(2)$ & $16(2)$ & $-7(2)$ & $-1(2)$ \\
C(1) & $47(7)$ & $51(7)$ & $18(5)$ & $-2(6)$ & 0 & \multicolumn{1}{c}{0} \\
C(2) & $40(5)$ & $45(5)$ & $34(4)$ & $1(4)$ & $-1(4)$ & $-2(4)$ \\
C(3) & $45(5)$ & $37(5)$ & $38(4)$ & $6(3)$ & $4(4)$ & $-2(4)$ \\
C(4) & $72(6)$ & $88(6)$ & $59(5)$ & $-5(5)$ & $-17(5)$ & $7(5)$ \\
C(5) & $91(7)$ & $90(7)$ & $50(5)$ & $-10(5)$ & $-2(5)$ & $-2(5)$ \\
C(6) & $132(8)$ & $83(7)$ & $81(7)$ & $-43(6)$ & $-29(6)$ & $11(5)$ \\
C(7) & $114(7)$ & $82(6)$ & $63(5)$ & $-9(6)$ & $-10(5)$ & $9(5)$ \\
C(8) & $79(6)$ & $88(7)$ & $99(7)$ & $-26(5)$ & $3(6)$ & $17(6)$ \\
C(9) & $105(7)$ & $111(8)$ & $57(5)$ & $11(6)$ & $-28(5)$ & $-2(5)$ \\
C(10) & $101(7)$ & $81(6)$ & $83(6)$ & $-5(6)$ & $0(6)$ & $32(5)$ \\
C(11) & $80(7)$ & $133(8)$ & $123(8)$ & $2(6)$ & $8(6)$ & $-9(7)$ \\
C(12) & $116(8)$ & $100(7)$ & $113(8)$ & $44(6)$ & $10(7)$ & $-17(6)$ \\
C(13) & $103(8)$ & $105(8)$ & $173(10)$ & $36(6)$ & $-4(7)$ & $32(7)$
\end{tabular}

${ }^{a}$ Der Temperaturfaktorexponent hat die Form: $-2 \pi^{2}\left(h^{2} a *{ }^{2} U_{11}\right.$ $\left.+\ldots+2 h k a * b * U_{12}\right)$.

Chlorpentamethyldisilan um, so erhält man nach gängiger Aufarbeitung das einfach disilanyl-substituierte Cyclopentadien 5 (Gl. (4)) in 60\%iger Aus-

TABELLE 5. H-Atomkoordinaten $\left(\times 10^{4}\right)$ und isotrope Auslenkungsparameter $\left(\AA^{2} \times 10^{3}\right)$ in 4

\begin{tabular}{lrlrl}
\hline Atom & \multicolumn{1}{l}{$x$} & \multicolumn{1}{l}{$y$} & $U$ \\
\hline H(2A) & 9150 & 6854 & 1533 & 80 \\
H(4A) & 8842 & 7979 & -325 & 80 \\
H(4B) & 8322 & 7399 & -535 & 80 \\
H(4C) & 8249 & 7737 & 124 & 80 \\
H(5A) & 10010 & 6892 & -650 & 80 \\
H(5B) & 9971 & 6157 & -340 & 80 \\
H(5C) & 9409 & 6380 & -827 & 80 \\
H(6A) & 7603 & 5402 & -155 & 80 \\
H(6B) & 7645 & 6179 & -367 & 80 \\
H(6C) & 8216 & 5650 & -579 & 80 \\
H(7A) & 8688 & 4834 & 796 & 80 \\
H(7B) & 9245 & 5073 & 310 & 80 \\
H(7C) & 9291 & 5332 & 999 & 80 \\
H(8A) & 7564 & 5757 & 1202 & 80 \\
H(8B) & 8084 & 6306 & 1471 & 80 \\
H(8C) & 7519 & 6534 & 989 & 80 \\
H(9A) & 10668 & 7478 & 3220 & 80 \\
H(9B) & 11198 & 6861 & 3200 & 80 \\
H(9C) & 11329 & 7526 & 2798 & 80 \\
H(10A) & 9523 & 6406 & 2885 & 80 \\
H(10B) & 9637 & 5923 & 2308 & 80 \\
H(10C) & 10109 & 5841 & 2896 & 80 \\
H(11A) & 12418 & 6479 & 1319 & 80 \\
H(11B) & 11953 & 7147 & 1337 & 80 \\
H(11C) & 12301 & 6873 & 1948 & 80 \\
H(12A) & 11346 & 5612 & 764 & 80 \\
H(12B) & 10601 & 5538 & 1049 & 80 \\
H(12C) & 10819 & 6230 & 719 & 80 \\
H(13A) & 11912 & 5180 & 1980 & 80 \\
H(13B) & 11773 & 5602 & 2590 & 80 \\
H(13C) & 11174 & 5168 & 2287 & 80 \\
\hline & & & & \\
\hline
\end{tabular}




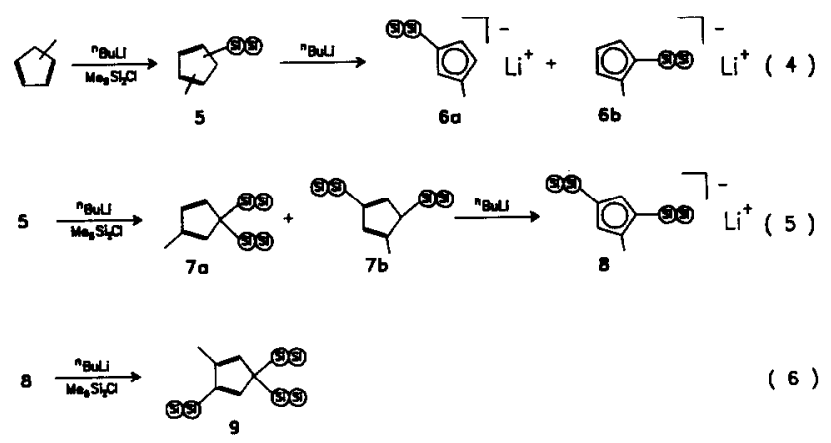

Schema 2.

beute als farbloses Öl, das kurzfristig an der Luft handhabbar ist. Insgesamt sind elf verschiedene Isomere von 5 möglich. NMR-spektroskopisch lassen sich nur vier Isomere nachweisen. Die Methylgruppe ist in allen vier Isomeren vinylständig, die Pentamethyldisilanyl-Gruppe ist in zwei Isomeren allylständig (5a, 5b) und in zwei anderen vinylständig. Das Hauptisomer ist 5a und steht zu den übrigen Isomeren in einem Verhältnis von etwa 3/1. Temperaturabhängige Untersuchungen zeigen, daß $\mathbf{5 a}$ und $\mathbf{5 b}$ durch silatrope Wanderung miteinander im Gleichgewicht stehen. Verbindung 5 ist wegen der in allen Isomeren vorhandenen Allyl-Wasserstoffatome deprotonierbar. Die Deprotonierung von 5 mit n-Butyllithium führt zur LithiumVerbindung 6 (Gl. (4)). Durch Entfernen des Lösungmittels erhält man 6 als farblosen, sehr luftempfindlichen Feststoff, der in THF gut und in unpolaren Lösungsmitteln schwer löslich ist. NMR-spektroskopisch lassen sich erwartungsgemä $\beta$ nur zwei Isomere nachweisen: die beiden Substituenten am Ring stehen entweder in 1,3-Position ( $\mathrm{ca}$. 85\%) oder in 1,2-Position (ca. $15 \%)$.

Die Umsetzung von 6 mit Chlorpentamethyldisilan liefert nach üblicher Aufarbeitung in 70\%iger Ausbeute das zweifach disilanyl-substituierte Cyclopentadien 7 als luftempfindliches gelbes Öl (Gl. (5)). NMRspektroskopisch lassen sich zwei Isomere nachweisen, die durch Silatropie miteinander in Gleichgewicht stehen. Das Hauptisomer ist 7a mit zwei allylischen Disilanylgruppen. Im ${ }^{1} \mathrm{H}-\mathrm{NMR}$-Spektrum bei $-80^{\circ} \mathrm{C}$ beobachtet man für die Protonen der $\mathrm{SiMe}_{2}$-Gruppen zwei Singuletts bei 0.29 und $0.30 \mathrm{ppm}$. Diese Aufspaltung läßt sich, wie auch bei Verbindung 2, durch unterschiedliche Wechselwirkungen mit dem Substituenten in 2-Stellung erklären (Diastereotopie). Aus sterischen Gründen sollte $\mathbf{7 b}$ das zweite beobachtete Isomer sein. Das Verhältnis von $\mathbf{7 a} \mathbf{z u} \mathbf{7 b}$ beträgt etwa $3 / 1$ und entspricht damit dem Isomerenverhältnis im ebenfalls zweifach disilanylsubstituierten 2. Allerdings lassen sich hier keine Isomere mit ausschließlich vinylständigen Silylgruppen nachweisen. Aufgrund des
Gleichgewichtes zwischen dem 5,5- und dem 2,5-Isomeren ist 7 quantitativ deprotonierbar. Die Umsetzung von $7 \mathrm{mit}{ }^{\mathrm{n}} \mathrm{BuLi}$ liefert die isomerenreine LithiumVerbindung 8 als luftempfindliches farbloses Pulver (Gl. (5)). Man findet im ${ }^{1}$ H-NMR-Spektrum ein Singulett für die ringständige Methylgruppe bei $2.17 \mathrm{ppm}$ und zwei Singuletts für die Ring-Wasserstoffatome bei 5.82 und $5.97 \mathrm{ppm}$. Diese Daten sind konsistent mit der erwarteten 1,2,4-Anordnung der Ring-Substituenten.

Die Umsetzung der Lithium-Verbindung 8 mit Chlorpentamethyldisilan liefert nach üblicher Aufarbeitung das dreifach disilanyl-substituierte Cyclopentadien 9 als farbloses, luftempfindliches öl in 63\%iger Ausbeute (Gl. (6)). NMR-spektroskopisch läßt sich erwartungsgemäß nur ein Isomer nachweisen. Temperaturabhängige NMR-Untersuchungen bis $100^{\circ} \mathrm{C}$ liefern keinen Hinweis für das Vorliegen einer Moleküldynamik.

Bei unseren Untersuchungen haben wir die Dimethylcyclopentadiene als Substrate aufgrund der Isomerenvielfalt ausgeschlossen. Bei den höher methylierten Cyclopentadienen wird die Anzahl der einzuführenden Disilanylgruppen durch die Anzahl der deprotonierbaren Ring-Wasserstoffatome begrenzt. Trimethylsilyl-Derivate des 1,2,4-Trimethylcyclopentadiens sind bisher in der Literatur noch unbekannt. Zuerst soll zu Vergleichszwecken untersucht werden, wieviele Trimethylsilyl-Gruppen in ein 1,2,4-Trimethylcyclopentadien eingebaut werden können. Dazu wird das 1,2,4-Trimethylcyclopentadien mit n-Butyllithium deprotoniert und mit Chlortrimethylsilan umgesetzt. Nach üblicher Aufarbeitung erhält man Verbindung 10 als farbloses luftempfindliches Öl in 70\%iger Ausbeute (Gl. (7)). Die zweite Silylierung auf gleichem Wege mit Chlortrimethylsilan gelingt problemlos, und man erhält 11 als farbloses hochviskoses Öl in 95\%iger Ausbeute (Gl. (7)). Verbindung 11 sollte aufgrund von silatropen Wanderungen aus einem Gemisch verschiedener Isomere bestehen. Die ${ }^{1} \mathrm{H}$-NMR-Untersuchungen zeigen eindeutig, daß zu 93\% 11a und $\mathrm{zu} 7 \%$ 11b vorliegt. Isomere mit allylständiger Methylgruppe lassen sich auch hier nicht nachweisen. Damit wird gezeigt, daß die Einführung von zwei Trimethylsilylgruppen in

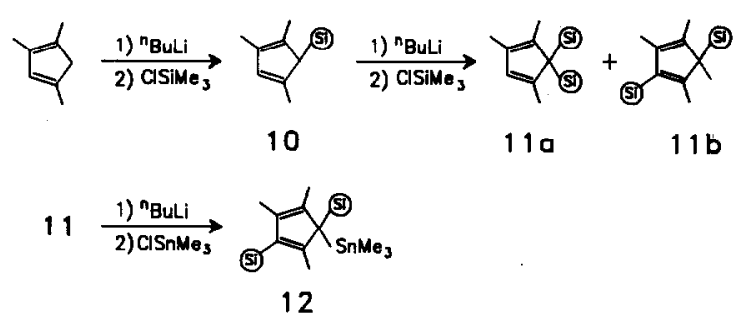

Schema 3. 
1,2,4-Trimethylcyclopentadien problemlos möglich ist. Außerdem stehen die Isomeren der zweifach silylierten Verbindung 11 miteinander im Gleichgewicht, so daß eine vollständige Deprotonierung möglich sein sollte.

Über die Deprotonierung von 11 mit n-Butyllithium ist die Einführung eines weiteren ElMe $_{3}$-Fragmentes möglich, wie wir am Beispiel der TrimethylstannylVerbindung 12 gezeigt haben. Man erhält 12 nach üblicher Aufarbeitung in 84\%iger Ausbeute (Gl. (8)). Verbindung 12 zeigt dynamisches Verhalten. Die geringe Aktivierungsenergie für die Wanderung der $\mathrm{SnMe}_{3}$-Gruppe führt dazu, daß die NMR-Spektren von 12 durch das Vorliegen gemittelter Resonanzen einen Gleichgewichtsprozess widerspiegeln. So liegt im ${ }^{29} \mathrm{Si}-\mathrm{NMR}$-Spektrum nur ein Signal für beide Silylgruppen vor, und im ${ }^{13} \mathrm{C}$-NMR-Spektrum sind die Signale der Si-substituierten Ring-Kohlenstoffatome stark verbreitert.

Diese Ergebnisse berechtigen dazu, im 1,2,4-Trimethylcyclopentadien die sukzessive Einführung von drei Pentamethyldisilanyl-Gruppen $\mathrm{zu}$ versuchen. Durch Umsetzung der entsprechenden LithiumVerbindung mit $\mathrm{Me}_{5} \mathrm{Si}_{2} \mathrm{Cl}$ erhält man nach üblicher Aufarbeitung in 80\%iger Ausbeute Verbindung 13 (Gl. (9)). NMR-spektroskopische Untersuchungen zeigen, $\mathrm{da} 13$ isomerenrein als Isomer mit allylständiger Pentamethyldisilanyl-Gruppe anfällt. Tempperaturabhängige Untersuchungen bis $100^{\circ} \mathrm{C}$ liefern keinen Hinweis auf eine vorliegende Moleküldynamik.

Die Einführung einer zweiten PentamethyldisilanylGruppe gelingt problemlos und führt nach üblicher Aufarbietung in 73\%iger Ausbeute zu Verbindung 14 (Gl. (9)). Im ${ }^{1}$ H-NMR-Spektrum von 14 findet man neben den Silylgruppen-Resonanzen drei Singuletts für vinylständige Methylgruppen und eine RingprotonenResonanz bei $7.14 \mathrm{ppm}$. Beide Disilanylgruppen befinden sich somit in Allylposition. Signale für andere Isomere sind nicht vorhanden, und temperaturabhängige Untersuchungen bis $100^{\circ} \mathrm{C}$ liefern keinen Hinweis auf eine Moleküldynamik. Verbindung 14 ist weder mit n-Butyllithium noch mit Kaliumhydrid deprotonierbar,

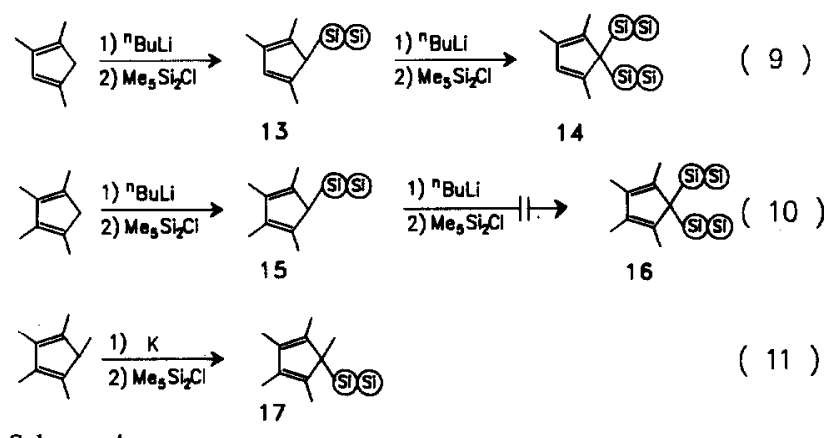

Schema 4. so daß auf diesem Weg eine dreifach silylierte Verbindung nicht zugänglich ist.

Durch Umsetzung von Tetramethylcyclopentadienyl-Lithium mit Chlorpentamethyldisilan erhält man in 66\%iger Ausbeute das einfach disilanylsubstituierte Tetramethylyclopentadien $\mathbf{1 5}$ als gelbes luftempfindliches Öl (Gl. (10)). Hinweise auf ein dynamisches Verhalten lassen sich nicht beobachten. Aufgrund des allylständigen Wasserstoffatoms ist 15 leicht deprotonierbar. Die Lithium-Verbindung von 15 kann zur Synthese entsprechender Cyclopentadienyl-MetallKomplexe genutzt werden [9]. Erstaunlicherweise ist aber die Synthese des zweifach silylierten Produktes 16 durch Umsetzung der Lithium-Verbindung von 15 mit Chlorpentamethyldisilan nicht gelungen (Gl. (10)).

Auch die Lithium-Verbindung des pentamethyl-substituierten Cyclopentadiens reagiert mit Chlorpentamethyldisilan in siedendem Toluol nicht. Die Umsetzung der entsprechenden Kalium-Verbindung führt problemlos zum gewünschten disilanyl-substituierten Pentamethylcyclopentadien 17 (Gl. (11)). Nach üblicher Aufarbeitung und destillativer Reinigung erhält man Verbindung 17 in 50\%iger Ausbeute als luftempfindliches gelbes Öl (Gl. (11)). Aufgrund der Äquivalenz aller Ringpositionen ist nur ein Isomer möglich. Die Dynamik der Verbindung zeigt sich in den temperaturabhängigen ${ }^{1} \mathrm{H}-\mathrm{NMR}$-Spektren: $\mathrm{Bis} 0^{\circ} \mathrm{C}$ findet man drei Resonanzen für die Ring-Methylgruppen als scharfe Singuletts. Mit steigender Temperatur verbreitern sich die Signale und koaleszieren. Bei $100^{\circ} \mathrm{C}$ liefern alle Ringmethylgruppen ein ausgemitteltes Singulett. Im Trimethylsilylpentamethylcyclopentadien fallen die Methylgruppen-Resonanzen bereits bei etwa $75^{\circ} \mathrm{C}$ zu einem scharfen Signal zusammen. Das bedeutet letztendlich eine geringere Wanderungsgeschwindigkeit der Pentamethyldisilanyl-Gruppe im Vergleich zur Trimethylsilylgruppe.

An zwei Beispielen soll die Reaktivität vinylständiger Protonen in Cyclopentadienyl-Systemen gegenüber dem Kaliumgraphit $\mathrm{C}_{8} \mathrm{~K}$ beschrieben werden. $\mathrm{C}_{8} \mathrm{~K}$ kann sowohl als starkes Reduktionsmittel als auch als starkes Deprotonierungsagens eingesetzt werden; es wird unter anderem zur Deprotonierung wenig $\mathrm{CH}$-acider Verbindungen, z.B. Ester und Nitrile, verwendet [10]. Deshalb sollte $\mathrm{C}_{8} \mathrm{~K}$ geeignet sein, $\mathrm{Cy}$ clopentadiene wie 4 und 14, die mit üblichen Deprotonierungsagentien nicht reagieren, zu deprotonieren.

Die Zugabe von $14 \mathrm{zu}$ einer Suspension von $\mathrm{C}_{8} \mathrm{~K}$ in THF und anschließende Alkylierung mit Methyliodid liefert nach gängiger Aufarbeitung und Kristallisieren aus Dichloromethan/Acetonitril farblose luftstabile Kristalle, die NMR-spektroskopisch als Verbindung 16 identifiziert werden (Gl. (12)). So findet man im ${ }^{1} \mathrm{H}$ NMR-Spektrum zwei Singuletts bei -0.26 und 0.32 


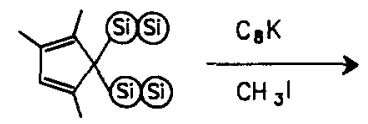

14

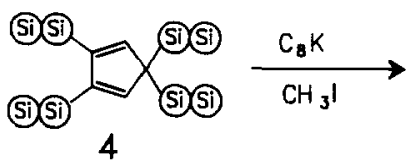

Schema 5.

ppm für Silylgruppen und zwei Singuletts bei 1.78 und $1.98 \mathrm{ppm}$ für vinylständige Methylgruppen.

Die Umsetzung von 4 mit $\mathrm{C}_{8} \mathrm{~K}$ und $\mathrm{CH}_{3} \mathrm{I}$ liefert nach gängiger Aufarbeitung nahezu quantitativ ein farbloses hochviskoses Öl, das NMR-spektroskopisch als Verbindung 9 identifiziert wird (Gl. (13)).

Offensichtlich verlaufen die Reaktionen von 4 und 14 mit $\mathrm{C}_{8} \mathrm{~K}$ unterschiedlich. Bei der Umsetzung von 14 mit $\mathrm{C}_{8} \mathrm{~K}$ tritt die gewünschte Deprotonierung ein, während bei der Umsetzung von $4 \mathrm{mit} \mathrm{C}_{8} \mathrm{~K}$ überraschenderweise eine $\mathrm{C}-\mathrm{Si}$-Bindung gespalten wird. Aus dem Ergebnis beider Reaktionen ergibt sich eine enorm hohe Stabilität der Si-Si-Bindung in diesen Systemen gegenüber starken Reduktionsmitteln.

Über die Komplexchemie der hier vorgestellten Disilanylcyclopentadiene wird an anderer Stelle berichtet [9].

\section{Experimenteller Teil}

Sämtliche Arbeiten wurden unter Ausschluß von Luft und Feuchtigkeit unter Argon ausgeführt; verwendete Geräte, Chemikalien und Lösungsmittel waren entsprechend vorbereitet. Schmelzpunkte: Büchi 510 Schmelzpunktbestimmungsapparatur; Probenröhrchen abgeschmolzen, Werte unkorrigiert. NMR-Spektren: Bruker AM 300: ${ }^{1} \mathrm{H}(300.1 \mathrm{MHz})$ ext. TMS; ${ }^{13} \mathrm{C}\left\{{ }^{1} \mathrm{H}\right\}$ (75.4 MHz) ext. TMS; ${ }^{29} \mathrm{Si}\left\{{ }^{1} \mathrm{H}\right\}(59.6 \mathrm{MHz})$ ext. TMS. Massenspektren: Varian 311 A $(60 \mathrm{eV}, 300 \mu$ A Emission), es sind nur charakteristische Fragmente angegeben. CHN-Analysen: Mikroanalytisches Laboratorium Beller (Göttingen) und Analytisches Labor der Fakultät für Chemie der Universität Bielefeld.

\subsection{Pentamethyldisilanyl-1,3-cyclopentadien (1)}

Man löst $16.01 \mathrm{~g}(242.2 \mathrm{mmol})$ frisch gecracktes $\mathrm{CpH}$ in $400 \mathrm{ml}$ THF, tropft bei $0^{\circ} \mathrm{C} 152 \mathrm{ml}{ }^{\mathrm{n}} \mathrm{BuLi}(1.60$ $\mathrm{m}$ in $\mathrm{n}$-Hexan, $243.2 \mathrm{mmol}$ ) langsam hinzu und rührt die farblose Suspension $16 \mathrm{~h}$ bei RT. Nach Zugabe von $40.80 \mathrm{~g}$ (244.6 mmol) $\mathrm{Me}_{5} \mathrm{Si}_{2} \mathrm{Cl}$ wird $4 \mathrm{~h}$ unter Rückfluß erhitzt. Man engt im Vakuum ein, versetzt den Rückstand mit $300 \mathrm{ml}$-Hexan, filtriert die festen
Bestandteile ab, wäscht zweimal mit $100 \mathrm{ml}$ n-Hexan nach und engt wieder im Vakuum ein. Eine anschließende Vakuumdestillation liefert Verbindung 1 als schwach gelbe Flüssigkeit.

Verbindung 1: Kp. (10 mbar): $70^{\circ} \mathrm{C}$ (Literatur: $\mathrm{Kp}$. (22 torr): $86^{\circ} \mathrm{C}$ [2]). Ausbeute $27.6 \mathrm{~g}(61 \%) .{ }^{1} \mathrm{H}-\mathrm{NMR}$ (Toluol- $d_{8}$ ) $-80^{\circ} \mathrm{C}: 1 \mathrm{a}(80 \%): \delta=-0.02(\mathrm{~s}, 6 \mathrm{H}$, $-\mathrm{SiMe}_{2}-$ ); 0.04 (s, 9H, $-\mathrm{SiMe}_{3}$ ); 3.30 (s, 1H, H-C(5)); $6.48(\mathrm{~m}, 2 \mathrm{H}, \mathrm{H}-\mathrm{C}(1,4))$; $6.63(\mathrm{~m}, 2 \mathrm{H}, \mathrm{H}-\mathrm{C}(2,3))$. 1b (15\%): $\delta=0.10$ (s, 9H, $\mathrm{SiMe}_{3}$ ); 0.16 (s, 6H, $\mathrm{SiMe}_{2}$ ); 2.79 (m, 2H, H-C(5)); 6.58(m, 2H, H-C (3,4)); $6.96(\mathrm{~m}$, 1H, H-C(2)). 1c (5\%): $\delta=0.25\left(\mathrm{~s}, 9 \mathrm{H}, \mathrm{SiMe}_{3}\right) ; 0.30(\mathrm{~s}$, $6 \mathrm{H}, \mathrm{SiMe}_{2}$ ); 2.74 (m, 2H, H-C(5)); 6.72 (m, H-C(vinyl)). $25^{\circ} \mathrm{C}: \delta=0.02\left(\mathrm{~s}, 6 \mathrm{H}, \mathrm{SiMe}_{2}\right) ; 0.08\left(\mathrm{~s}, 9 \mathrm{H}, \mathrm{SiMe}_{3}\right)$; 3.3-3.5 (breites s, $1 \mathrm{H}, \mathrm{H}-\mathrm{C}(5)) ; 6.65-6.50(\mathrm{~m}, 4 \mathrm{H}, \mathrm{Cp}$ H). $100^{\circ} \mathrm{C}: \delta=-0.06\left(\mathrm{~s}, 6 \mathrm{H}, \mathrm{SiMe}_{2}\right) ; 0.00(\mathrm{~s}, 9 \mathrm{H}$, $\mathrm{SiMe}_{3}$ ); 5.62-5.93 (breites $\mathrm{s}, 5 \mathrm{H}, \mathrm{Cp}-\mathrm{H}$ ). ${ }^{13} \mathrm{C}-\mathrm{NMR}$ $\left(\mathrm{CDCl}_{3}\right)-50^{\circ} \mathrm{C}: \quad$ 1a: $\delta=-4.26 \quad\left(\mathrm{SiMe}_{3}\right) ; \quad-1.65$ $\left(\mathrm{SiMe}_{2}\right) ; 50.45$ (C(5)); 129.69 (C(2,3)); 133.32 (C(1,4)). 1b: $\delta=-3.47 \quad\left(-\mathrm{SiMe}_{3}\right) ;-2.26 \quad\left(-\mathrm{SiMe}_{2}-\right) ; 45.63$ (C(5)); 133.03 (C(4)); $137.61(\mathrm{C}(3)) ; 142.32(\mathrm{C}(3)) ; 146.55$ (C(1)). 1c: $\delta=38.32$ (C(5)); 135.80; 138.03; 142.32; (C(vinyl)); $156.00(\mathrm{C}(2)) .{ }^{29}$ Si-NMR $\left(\mathrm{CDCl}_{3}\right): 1 \mathrm{a}: \delta=$ -18.3 $\left(\mathrm{SiMe}_{3}\right),-14.7\left(\mathrm{SiMe}_{2}\right) .1 \mathrm{~b} / 1 \mathrm{c}:-27.8\left(\mathrm{SiMe}_{2}\right)$, $-19.6\left(\mathrm{SiMe}_{3}\right)$.

\subsection{Bis-(pentamethyldisilanyl)-1,3-cyclopentadien (2)}

Man löst $15.70 \mathrm{~g}(79.9 \mathrm{mmol}) 1$ in $150 \mathrm{ml} \mathrm{THF}$, tropft bei $0^{\circ} \mathrm{C} 50 \mathrm{ml}{ }^{\mathrm{n}} \mathrm{BuLi}(1.60 \mathrm{~m}$ in $\mathrm{n}-\mathrm{Hexan}, 80.0$ mmol) langsam hinzu und rührt $3 \mathrm{~d}$ bei RT, bis eine klare Lösung entstanden ist. Danach werden $13.35 \mathrm{~g}$ $(80.0 \mathrm{mmol}) \mathrm{Me}_{5} \mathrm{Si}_{2} \mathrm{Cl}$ zugegeben und $4 \mathrm{~h}$ unter Rückfluß erhitzt. Nach üblicher Aufarbeitung liefert eine Vakuumdestillation Verbindung 2 als gelbe Flüssigkeit.

Verbindung 2: Kp. (0.2 mbar): $65-108^{\circ} \mathrm{C}$. Ausbeute $19.8 \mathrm{~g}(76 \%)$. ${ }^{1} \mathrm{H}-\mathrm{NMR}$ (Toluol $-d_{8}$ ) $-80^{\circ} \mathrm{C}: 2 \mathrm{a}: \delta=$ -0.01 (s, 18H, $\left.\mathrm{SiMe}_{3}\right) ; 0.32$ (s, 12H, $\left.\mathrm{SiMe}_{2}\right) ; 6.33$ (m, $2 \mathrm{H}, \mathrm{H}-\mathrm{C}(1,4)) ; 6.61(\mathrm{~m}, 2 \mathrm{H} \mathrm{H}-\mathrm{C}(2,3)) .2 \mathrm{~b}: \delta=0.09(\mathrm{~s}$, $\left.9 \mathrm{H}, \mathrm{SiMe}_{3}\right) ; 0.15$ (s, 6H, C(5)SiMe $) ; 0.24$ (s, 9H, $\mathrm{SiMe}_{3}$ ); 3.50(b) (s, 1H, H-C(5)); 6.81(b) (m, 3H, H$\mathrm{C}($ vinyl)). andere Isomere: $\delta=2.95(\mathrm{~b}) ; 3.22(\mathrm{~b})(\mathrm{s}, 2 \mathrm{H}$, H-C(5)). $100^{\circ} \mathrm{C}: 2 \mathrm{a} / 2 \mathrm{~b}: \delta=0.04(\mathrm{~b})\left(\mathrm{s}, 36 \mathrm{H}, \mathrm{SiMe}_{3}\right.$; $0.24(\mathrm{~b})$ (s, 24H, $\mathrm{SiMe}_{2}$ ), 5.0-5.5 (sehr breites s, $1 \mathrm{H}$, $\mathrm{H}-\mathrm{Cp})$; 6.61 (s, 3H, H-C(vinyl)). andere Isomere: 0.21 ; 0.23 (s, Si-Me); $2.99(\mathrm{~b}) ; 3.11(\mathrm{~b})(\mathrm{s}, 2 \mathrm{H}, \mathrm{H}-\mathrm{C}(5)) .{ }^{13} \mathrm{C}-$ NMR $\left(\mathrm{CDCl}_{3}\right):-50^{\circ} \mathrm{C}: \delta=-4.64 ;-4.54 ;-3.60$; $-3.47 ;-2.04 ;-1.69 ;-1.14 ;-0.86 ; 1.85$ (Si-Me). 2a: $\delta=54.83(\mathrm{C}(5)) ; 130.55(\mathrm{C}(2,3)) ; 137.2(\mathrm{C}(1,4)) .2 \mathrm{~b}$ : $\delta=52.87(\mathrm{C}(5)) ; 133.21 ; 133.75(\mathrm{C}(3,4)) ; 141.51(\mathrm{C}(2))$; $142.24(\mathrm{C}(1))$. andere Isomere: $\delta=50.30(\mathrm{C}(5)) ; 142.06$ (C(vinyl)); 151.93 (C(vinyl)). ${ }^{29} \mathrm{Si}-\mathrm{NMR}\left(\mathrm{CDCl}_{3}\right): \delta=$ -28.6 ; -28.1 (C(vinyl)-Si); -19.5 ; -18.2 : -17.6 (-Si$\left.\mathrm{Me}_{3}\right) ;-15.7 ;-15.4$ (C(5)-Si). MS: $m / z(\%)=326$ 
$\left(4.0\left((M)^{+}, 253(16.6)\left(M-\mathrm{SiMe}_{3}\right)^{+}, 165 \quad\right.\right.$ (32.8) $\left(\mathrm{C}_{5} \mathrm{H}_{4} \mathrm{Si}_{2} \mathrm{Me}_{3}\right)^{+}, \quad 131 \quad(19.4) \quad\left(\mathrm{Si}_{2} \mathrm{Me}_{5}\right)^{+}, 73 \quad$ (100) $\left(\mathrm{SiMe}_{3}\right)^{+}$. Gef.: C, 54.29; H, 10.11. $\mathrm{C}_{15} \mathrm{H}_{34} \mathrm{Si}_{4}$ (326.78) ber.: $\mathrm{C}, 55.13 ; \mathrm{H}, 10.49 \%$.

\subsection{2,5,5-Tris-(pentamethyldisilanyl)-1,3-cyclopentadien} (3)

Man löst $15.03 \mathrm{~g}(46.0 \mathrm{mmol}) 2$ in $130 \mathrm{ml}$ THF, tropft bei $0^{\circ} \mathrm{C} 29 \mathrm{ml}{ }^{n} \mathrm{BuLi}(1.60 \mathrm{~m}$ in $\mathrm{n}$-Hexan, 46.4 mmol) langsam hinzu und rührt $16 \mathrm{~h}$ bei RT, bis eine klare gelbe Lösung entstanden ist. Nach der Zugabe von $7.68 \mathrm{~g}$ ( $46.4 \mathrm{mmol}) \mathrm{Me}_{5} \mathrm{Si}_{2} \mathrm{Cl}$ wird $6 \mathrm{~h}$ bei $\mathrm{RT}$ gerührt. Nach üblicher Aufarbeitung liefert eine Vakuumdestillation Verbindung 3 als gelbe Flüssigkeit.

Verbindung 3: Kp. $(0.1 \mathrm{mbar}): 125-136^{\circ} \mathrm{C}$. Ausbeute $15.5 \mathrm{~g}(74 \%)$. ' $\mathrm{H}-\mathrm{NMR}\left(\mathrm{CDCl}_{3}\right) \delta=-0.10(\mathrm{~s}, 18 \mathrm{H}$, $\mathrm{SiMe}_{3}$ ); 0.09 (s, 9H, SiMe ${ }_{3}$ ); 0.14; 0.15 (s, 6H, C(5)$\mathrm{SiMe}_{2}$ ); 0.21 (s, 6H, C(2)-SiMe $\left.{ }_{2}\right) ; 6.58$ (m, 1H, H-C(3)); $6.72(\mathrm{~m}, 2 \mathrm{H}, \mathrm{H}-\mathrm{C}(1,4))$. ${ }^{1} \mathrm{H}-\mathrm{NMR}$ (Toluol- $\left.d_{8}\right)$ : $0^{\circ} \mathrm{C}$ : $\delta=0.01\left(\mathrm{~s}, 18 \mathrm{H}, \mathrm{SiMe}_{3}\right) ; 0.21 ; 0.22 ; 0.23$ (s, zusammen $21 \mathrm{H}, \mathrm{Si}-\mathrm{Me}) ; 6.58(\mathrm{~m}, 1 \mathrm{H}, \mathrm{H}-\mathrm{C}(3)) ; 6.80\left(\mathrm{~d},{ }^{3} \mathrm{~J}=4.3 \mathrm{~Hz}\right.$, $1 \mathrm{H}, \mathrm{H}-\mathrm{C}(4)) ; 6.85$ (s, $1 \mathrm{H}, \mathrm{H}-\mathrm{C}(1)) .100^{\circ} \mathrm{C}: \delta=0.05$ (s, $\left.27 \mathrm{H}, \mathrm{SiMe}_{3}\right) ; 0.21$ (s, 18H, $\left.\mathrm{SiMe}_{2}\right) ; 6.61(\mathrm{~s}, 2 \mathrm{H}, \mathrm{H}-$ C(vinyl)); 6.84 (s, $1 \mathrm{H}, \mathrm{H}$-C(vinyl)). ${ }^{13} \mathrm{C}$-NMR $\left(\mathrm{CDCl}_{3}\right)$ : $\delta=-3.04 ;-1.51 ;-0.59$ (Si-Me); 57.57 (C(5)); 134.90 (C(3)); 137.32 (C(4)); $141.81(\mathrm{C}(2)) ; 146.09(\mathrm{C}(1)) .{ }^{29} \mathrm{Si}-$ NMR $\left(\mathrm{CDCl}_{3}\right): \delta=-29.2(\mathrm{Si}-\mathrm{C}(2)) ;-19.4(\mathrm{Si}-\mathrm{C}(5))$; $-17.4\left(\mathrm{SiMe}_{3}\right)$. MS: $m / z(\%)=456(1.8)(M)^{+} ; 383$ (9.7) $\left(M-\mathrm{SiMe}_{3}\right)^{+} ; 295$ (22.0); 237 (15.2); 131 (16.0) $\left(\mathrm{Me}_{5} \mathrm{Si}_{2}\right)^{+} ; 73(100)\left(\mathrm{Me}_{3} \mathrm{Si}\right)^{+}$. Gef.: C, 52.48; H, 10.32; $\mathrm{C}_{20} \mathrm{H}_{48} \mathrm{Si}_{6}$ (457.11) ber.: $\mathrm{C}, 52.55 ; \mathrm{H}, 10.58 \%$.

3.4. 2,3,5,5-Tetrakis-(pentamethyldisilanyl)-1,3-cyclopentadien (4)

Man löst $10.06 \mathrm{~g}(22.0 \mathrm{mmol}) 3 \mathrm{in} 150 \mathrm{ml}$ THF, tropft bei $0^{\circ} \mathrm{C} 20 \mathrm{ml}{ }^{n} \mathrm{BuLi}(1.60 \mathrm{~m}$ in $\mathrm{n}-\mathrm{Hexan}, 32$ mmol) langsam hinzu und rührt die braune Lösung $3 \mathrm{~d}$ bei RT. Nach der Zugabe von $5.34 \mathrm{~g}(32 \mathrm{mmol})$ $\mathrm{Me}_{5} \mathrm{Si}_{2} \mathrm{Cl}$ wird noch $12 \mathrm{~h}$ bei $\mathrm{RT}$ gerührt. Übliche Aufarbeitung und Kristallisation aus $\mathrm{CH}_{2} \mathrm{Cl}_{2} / \mathrm{CH}_{3} \mathrm{CN}$ liefert Verbindung 4 als nadelförmige farblose Kristalle.

Verbindung 4: Schmp. $75.8^{\circ} \mathrm{C}$. Ausbeute $5.05 \mathrm{~g}$ (50\%). ${ }^{1} \mathrm{H}-\mathrm{NMR}\left(\mathrm{CDCl}_{3}\right) \delta=-0.09(\mathrm{~s}, 18 \mathrm{H}, \mathrm{C}(5)-\mathrm{Si}$ $-\mathrm{SiMe}_{3}$ ); 0.09 (s, 12H, C(5)-SiMe ${ }_{2}$; 0.11 (s, 18H, $\mathrm{C}(2,3)-\mathrm{Si}-\mathrm{SiMe}_{3}$ ); 0.25 (s, 12H, C(2,3)-SiMe 2 ); 6.85 (s, $2 \mathrm{H}, \mathrm{H}-\mathrm{C}(1,4)) .{ }^{13} \mathrm{C}-\mathrm{NMR}\left(\mathrm{CDCl}_{3}\right): \delta=-2.01 ;-1.18$; -0.93 (Si-Me); 59.02 (C(5)); $145.7(\mathrm{C}(2,3)) ; 149.51$ $(\mathrm{C}(1,4)) .{ }^{29} \mathrm{Si}-\mathrm{NMR}\left(\mathrm{CDCl}_{3}\right): \delta=-26.6(\mathrm{C}(2,3)-\mathrm{Si})$; $-18.8 ;-18.6\left(-\mathrm{SiMe}_{3}\right) ;-17.2(\mathrm{C}(5)-\mathrm{Si})$. MS; $m / z$ $(\%)=586(0.14)(M)^{+} ; 513(5.6)\left(M-\mathrm{SiMe}_{3}\right)^{+} ; 425$ (6.1); $366(7.7) ; 131(19.9)\left(\mathrm{Me}_{5} \mathrm{Si}_{2}\right)^{+} ; 73(100)\left(\mathrm{Me}_{3} \mathrm{Si}\right)^{+}$.

\subsection{Pentamethyldisilanyl-(methyl)-1,3-cyclopentadien (5)}

Man löst $20.0 \mathrm{~g}(250 \mathrm{mmol})$ frisch gecracktes $\mathrm{MeCpH}$ in $450 \mathrm{ml} \mathrm{THF}$, tropft bei $0^{\circ} \mathrm{C} 193 \mathrm{ml}(1.30 \mathrm{~m}$ in
n-Hexan, $251 \mathrm{mmol}){ }^{\mathrm{n}} \mathrm{BuLi}$ langsam hinzu und rührt die Suspension 16 h bei RT. Nach Zugabe von $41.7 \mathrm{~g}$ (250 mmol) $\mathrm{Me}_{5} \mathrm{Si}_{2} \mathrm{Cl}$ wird $3 \mathrm{~h}$ unter Rückfluß erhitzt und weitere $16 \mathrm{~h}$ bei RT gerührt. Übliche Aufarbeitung und Destillation im Vakuum liefert 5 als gelbe Flüssigkeit.

Verbindung 5: Kp. (5 mbar): $40-45^{\circ} \mathrm{C}$. Ausbeute $31.8 \mathrm{~g}(60 \%) .{ }^{1} \mathrm{H}-\mathrm{NMR}$ (Toluol- $d_{8}$ ): $-80^{\circ} \mathrm{C} \mathrm{5a:} \delta=0.01$ (s, 9H, $\mathrm{SIMe}_{3}$ ); 0.03 (s, 6H, SiMe $) ; 2.03$ (s, 3H, $\mathrm{CH}_{3}$ ); 3.21 (s, 1H, C(5)-H); 6.06 (s, $1 \mathrm{H}, \mathrm{C}($ vinyl)-H); 6.45 (s, $2 \mathrm{H}$, C(vinyl)-H). Andere Isomere: $\delta=2.60 ; 2.84 ; 2.94$ (s, jeweils $2 \mathrm{H}, \mathrm{C}(5)-\mathrm{H}) \cdot 100^{\circ} \mathrm{C}: 5 \mathrm{a} / 5 \mathrm{~b}: \delta=0.00(\mathrm{~s}, 9 \mathrm{H}$, $\left.\mathrm{SiMe}_{3}\right) ; 0.01$ (s, 6H, SiMe $)$ ); 1.99 (s, 3H, $\left.\mathrm{CH}_{3}\right) ; 4.5-5.67$ (s(breit), Cp-H); 5.9 (s(breit); 1H, C(vinyl)-H); 6.61, 6.62 (s, jeweils $1 \mathrm{H}, \mathrm{C}($ vinyl)-H). Andere Isomere: 0.02 ; $0.06 ; 0.07 ; 0.19$ (s, Si-Me); 1.89; 1.93 (s, jeweils $3 \mathrm{H}$, $\left.\mathrm{CH}_{3}\right) ; 2.78 ; 2.91(\mathrm{~s}$, jeweils $2 \mathrm{H}, \mathrm{C}(5)-\mathrm{H}) .{ }^{13} \mathrm{C}-\mathrm{NMR}$ $\left(\mathrm{CDCl}_{3}\right)-50^{\circ} \mathrm{C}: \delta=-4.64 ;-3.65 ;-2.74 ;-2.31$ (SiMe). 5a: $\delta=15.24$ (C(2)-Me); 49.65 (C(5)); 128.16; $132.77 ; 134.21(\mathrm{C}(1,2,4) ; 139.75(\mathrm{C}(2))$. Andere Isomere: $14.90 ; 16.30 ; 16.88$ (C-Me); 45.30; 52.46 (C(5)). ${ }^{29} \mathrm{Si}-\mathrm{NMR}\left(\mathrm{CDCl}_{3}\right): \delta=-28.1$ (C(vinyl)Si); -19.4 ; $-18.5 ;-18.0\left(\mathrm{SiMe}_{3}\right) ;-13.9$ (C(5)Si). MS: $m / z(\%)$ $=210(14.2)(M)^{+} ; 196(12.7)\left(M-\mathrm{CH}_{2}\right)^{+}, 73(100)$ $\left(\mathrm{SiMe}_{3}\right.$ ). Gef.: C, 62.72; H, 10.40. $\mathrm{C}_{11} \mathrm{H}_{22} \mathrm{Si}_{2}(210.47)$ ber.: $\mathrm{C}, 62.78 ; \mathrm{H}, 10.54 \%$.

\subsection{Pentamethyldisilanyl-(methyl)-cyclopentadienyl- Lithium (6)}

Man löst $337 \mathrm{mg}$ ( $1.60 \mathrm{mmol}) 5 \mathrm{in} 20 \mathrm{ml}$ THF, tropft bei $0^{\circ} \mathrm{C} 1 \mathrm{ml}(1.60 \mathrm{~m}$ in $\mathrm{n}-\mathrm{Hexan}, 1.60 \mathrm{mmol}){ }^{\mathrm{n}} \mathrm{BuLi}$ langsam hinzu und rührt $16 \mathrm{~h}$ bei RT. Das Lösungsmittel wird im Vakuum entfernt, und man erhält 6 als farbloses Pulver.

Verbindung 6: ${ }^{1} \mathrm{H}-\mathrm{NMR}$ (THF- $d_{8}$ ): $\delta=\mathbf{0 . 1 0}$ (s, 9H, $\mathrm{SiMe}_{3}$ ); 0.24 (s, 6H, $\mathrm{SiMe}_{2}, 1,3-$ Isomer); 0.31 (s, 6H, $\mathrm{SiMe}_{2}, 1,2$-Isomer); 2.18 (s, 3H, Cp-Me, 1,3-Isomer); 2.24 (s, 3H, Cp-Me, 1,2-Isomer); 5.71 (d, ${ }^{4} J=2.0 \mathrm{~Hz}$, $2 \mathrm{H}, \mathrm{Cp}-\mathrm{H}, 1,3-\mathrm{Isomer}) ; 5.81\left(\mathrm{t},{ }^{4} J=2.0 \mathrm{~Hz}, 1 \mathrm{H}, \mathrm{Cp}-\mathrm{H}\right.$, 1,3-Isomer).

\subsection{Bis-(pentamethyldisilanyl)-methyl-1,3-cyclopentadien} (7)

Man löst $16.8 \mathrm{~g}(80 \mathrm{mmol}) 5$ in $350 \mathrm{ml}$ THF, tropft bei $0^{\circ} \mathrm{C} 50 \mathrm{ml}(1.60 \mathrm{~m}$ in $\mathrm{n}$-Hexan, $80 \mathrm{mmol}){ }^{n} \mathrm{BuLi}$ langsam hinzu und rührt $16 \mathrm{~h}$ bei RT. Zu der klaren gelben Lösung gibt man $13.5 \mathrm{~g}(81 \mathrm{mmol}) \mathrm{Me}_{5} \mathrm{Si}_{2} \mathrm{Cl}$ und erhitzt $4 \mathrm{~h}$ unter Rückfluß. Nach üblicher Aufarbeitung und Destillation im Vakuum erhält man 7 als schwach gelbe Flüssigkeit.

Verbindung 7: Kp. $(0.1 \mathrm{mbar}): 82-86^{\circ} \mathrm{C}$. Ausbeute $19.2 \mathrm{~g}(70 \%)$. ${ }^{1} \mathrm{H}-\mathrm{NMR}$ (Toluol- $d_{8}$ ): $-80^{\circ} \mathrm{C}: 7 \mathrm{a}: \delta=$ $-0.03\left(\mathrm{~s}, 18 \mathrm{H}, \mathrm{SiMe}_{3}\right) ; 0.29 ; 0.30$ (s, jeweils $6 \mathrm{H}, \mathrm{SiMe}_{2}$ ); $2.01\left(\mathrm{~s}, 3 \mathrm{H}, \mathrm{CH}_{3}\right) ; 5.94 ; 6.23,6.38(\mathrm{~s}$, jeweils $1 \mathrm{H}$, 
C(vinyl)-H). $7 \mathrm{~b}: \delta=0.05$ (s, $\left.18 \mathrm{H}, \mathrm{SiMe}_{3}\right) ; 0.20$ (s, 6H, $\left.\mathrm{C}(5) \mathrm{SiMe}_{2}\right) ; 0.39$ (s, 6H, C(2)-H); $2.16\left(\mathrm{~s}, 3 \mathrm{H}, \mathrm{CH}_{3}\right.$ ); 3.33 (s, $1 \mathrm{H}, \mathrm{C}(5)-\mathrm{H}), 6.18 ; 6.80$ (s, jeweils $1 \mathrm{H}, \mathrm{C}($ vinyl)H). $100^{\circ} \mathrm{C}: 7 \mathrm{a} / 7 \mathrm{~b}: \delta=0.00\left(\mathrm{~s}, 18 \mathrm{H}, \mathrm{SiMe}_{3}\right) ; 0.09$ (s, 12H, $\mathrm{SiMe}_{3}$ ); 0.24; 0.32 (s, jeweils 12h, $\mathrm{SiMe}_{2}$ ); 2.04; 2.14 (s, jeweils $3 \mathrm{H}, \mathrm{CH}_{3}$ ); 3.3-3.8 (s(breit), $1 \mathrm{H}$, (C(5)$\mathrm{H}$ ); 5.7-6.0 (s(breit), 1H, C(3)-H); 6.22; 6.43; 6.65; 6.70 (s, jeweils $1 \mathrm{H}, \mathrm{C}$ (vinyl)-H). ${ }^{13} \mathrm{C}-\mathrm{NMR}\left(\mathrm{CDCl}_{3}\right)-50^{\circ} \mathrm{C}$ : $\delta=-4.42 ;-4.15 ;-3.38 ;-3.12 ;-2.12 ;-1.80$; -1.17 (Si-Me). 7a: 15.23 (C(2)-Me); 53.28 (C(5)); $132.51 ; 133.26 ; 137.54(\mathrm{C}(1,3,4)) ; 139.98(\mathrm{C}(2)) .7 \mathrm{~b}: \delta=$ 16.75 (C(3)-Me); 51.23 (C(5)); 128.85 (C(3)); 142.92; 144.07 (C(2,4)); 144.71 (C(1)). ${ }^{29} \mathrm{Si}-\mathrm{NMR}\left(\mathrm{CDCl}_{3}\right)$ : -28.7 (C(vinyl)Si); $-19.3 ;-18.6 ;-18.1 ;-17.5$ $\left(\mathrm{SiMe}_{3}\right) ;-15.5 ;-14.8(\mathrm{C}(5) \mathrm{Si})$. MS: $m / z(\%)=339$ (1.0) $(M-\mathrm{H})^{+} ; 325(3.0)(M-\mathrm{Me})^{+} ; 267$ (46.4) $(M-$ $\left.\mathrm{SiMe}_{3}\right)^{+} ; 209(11.6)\left(M-\mathrm{Si}_{2} \mathrm{Me}_{5}\right)^{+} ; 194(8.7)(M-$ $\left.\mathrm{Si}_{2} \mathrm{Me}_{6}\right)^{+}, 73(100)\left(\mathrm{SiMe}_{3}\right)^{+}$. Gef.: C, 56.75; H, 10.73. $\mathrm{C}_{16} \mathrm{H}_{36} \mathrm{Si}_{4}$ (340.81) ber.: C, 56.39; H, $10.65 \%$.

\subsection{1,3-Bis(pentamethyldisilanyl)-4-methyl-cyclopenta-} dienyl-Lithium (8)

Man löst $545 \mathrm{mg}$ ( $1.60 \mathrm{mmol}) 7 \mathrm{in} 20 \mathrm{ml}$ THF, tropft bei $0^{\circ} \mathrm{C} 1 \mathrm{ml}(1.60 \mathrm{~m}$ in $\mathrm{n}-\mathrm{Hexan}, 1.60 \mathrm{mmol}){ }^{\mathrm{n}} \mathrm{BuLi}$ langsam hinzu und rührt $16 \mathrm{~h}$ bei RT. Das Lösungsmittel wird im Vakuum entfernt, und man erhält 8 als farbloses Pulver.

Verbindung 8: ${ }^{1} \mathrm{H}-\mathrm{NMR}$ (THF- $\left.d_{8}\right): \delta=-0.06(\mathrm{~s}$, 18H, SiMe ${ }_{3}$ ); 0.13 (s, 6H, SiMe $)_{2}$ ); 0.20 (s, 6H, SiMe ${ }_{2}$ ); 2.16 (s, 3H, Cp-Me); 5.82 (s, 1H, Cp-H); 5.9 (s, 1H, $\mathrm{Cp}-\mathrm{H})$.

\subsection{2,2,5-Tris-(pentamethyldisilanyl)-3-methyl-1,3-} cyclopentadien (9)

(a) Man löst $4.00 \mathrm{~g}(11.7 \mathrm{mmol}) 7$ in $100 \mathrm{ml} \mathrm{THF}$, tropft bei $0^{\circ} \mathrm{C} 8 \mathrm{ml}(1.6 \mathrm{~m}$ in $\mathrm{n}$-Hexan, $12.8 \mathrm{mmol})$ ${ }^{n} \mathrm{BuLi}$ langsam hinzu und rïhrt $16 \mathrm{~h}$ bei RT. $\mathrm{Zu}$ der farblosen Lösung gibt man $2.20 \mathrm{~g}(13.0 \mathrm{mmol})$ $\mathrm{Me}_{5} \mathrm{Si}_{2} \mathrm{Cl}$, erhitzt $6 \mathrm{~h}$ unter Rückfluß und läßt weitere $16 \mathrm{~h}$ rühren. Übliche Aufarbeitung und Destillation im Vakuum liefert $3.50 \mathrm{~g}(63 \%) 9$ als farbloses Öl.

(b) $\mathrm{Zu}$ einer Suspension von $410 \mathrm{mg}(3.00 \mathrm{mmol})$ $\mathrm{C}_{8} \mathrm{~K}$ in $20 \mathrm{ml}$ THF gibt man eine Lösung von $890 \mathrm{mg}$ (1.50 mmol) 4 in $10 \mathrm{ml}$ THF und rührt $2 \mathrm{~h}$ bei RT. Nach der Zugabe von $500 \mathrm{mg}$ ( $3.40 \mathrm{mmol}) \mathrm{CH}_{3} \mathrm{I}$ wird weitere $16 \mathrm{~h}$ gerührt. Man engt im Vakuum ein, fügt 50 $\mathrm{ml}$ n-Hexan hinzu, filtriert vom unlöslichen Rückstand über Kieselgel $a b$ und engt im Vackuum ein. Man erhält $700 \mathrm{mg}$ (95\%) 9 als farbloses Öl.

Verbindung 9: Kp. (0.1 mbar): $103-110^{\circ} \mathrm{C} .{ }^{1} \mathrm{H}-\mathrm{NMR}$ (Toluol $\left.-d_{8}\right)$ : $-80^{\circ} \mathrm{C}: \delta=-0.05\left(\mathrm{~s}, 18 \mathrm{H}, \mathrm{SiMe}_{3}\right) ; 0.22$ (s, 12H, C(5)SiMe $)_{2}$; 0.26 (s, 9H, $\mathrm{SiMe}_{3}$ ); 0.38 (s, 6H, $\mathrm{C}(2) \mathrm{SiMe}_{2}$ ); 2.11 (s, 3H, C(3)-Me); 6.04 (s, 1H, C(4)-H); $6.83(\mathrm{~s}, 1 \mathrm{H}, \mathrm{C}(1)-\mathrm{H}) .100^{\circ} \mathrm{C}: \delta=-0.01 ; 0.06 ; 0.07 ; 0.10$;
$0.20 ; 0.27 ; 0.30$ (7s, zusammen $45 \mathrm{H}, \mathrm{Si}-\mathrm{Me}) ; 2.15$ (s, 3H, $\mathrm{C}(3)-\mathrm{Me}) ; 6.23$ (s, 1H, C(4)-H); 6.77 (s, 1H, C(1)-H). ${ }^{13} \mathrm{C}-\mathrm{NMR}\left(\mathrm{CDCl}_{3}\right)-50^{\circ} \mathrm{C}:-3.37 ;-3.11 ;-2.52$; $-1.76 ;-1.38 ;-1.15$ (Si-Me); 16.52 (C(3)-Me); 55.00 (C(5)); 133.30 (C(4)); 142.07; 144.31 (C(2,3)); 147.92 (C(1)). ${ }^{29} \mathrm{Si}-\mathrm{NMR}\left(\mathrm{CDCl}_{3}\right): \delta=-29.4(\mathrm{C}(2) \mathrm{Si}) ;-19.0$; $-17.9 ;-16.6(\mathrm{MeSi})$. MS: $m / z(\%)=470(1.0)(M)^{+}$, 396 (33.6) $\left(M-\mathrm{SiMe}_{3}\right)^{+} ; 309$ (32.2); 251 (18.0); 131 (25.0) $\left(\mathrm{Me}_{5} \mathrm{Si}_{2}\right)^{+} ; 73(100)\left(\mathrm{SiMe}_{3}\right)^{+}$. Gef.: C, 53.12; $\mathrm{H}$, 10.50. $\mathrm{C}_{21} \mathrm{H}_{50} \mathrm{Si}_{6}(471.14)$ ber: $\mathrm{C}, 53.53 ; \mathrm{H}, 10.70 \%$.

\subsection{5-Trimethylsilyl-1,2,4-trimethyl-1,3-cyclopentadien (10)}

Man löst $10.01 \mathrm{~g}$ (92.5 mmol) 1,2,4-Trimethyl-1,3cyclopentadien in $300 \mathrm{ml}$ THF, tropft bei RT $64 \mathrm{ml}$ (1.60 $\mathrm{m}$ in $\mathrm{n}$-Hexan, $96.0 \mathrm{mmol}$ ) ${ }^{\mathrm{n}} \mathrm{BuLi}$ langsam hinzu und rührt die Suspension $12 \mathrm{~h}$. Danach werden $10.52 \mathrm{~g}$ $(97.0 \mathrm{mmol}) \mathrm{Me}_{3} \mathrm{SiCl}$ in $100 \mathrm{ml} \mathrm{THF}$ zugetropft. Nach $48 \mathrm{~h}$ Rühren bei RT erhält man eine klare gelbe Lösung. Nach Einengen auf ca. $80 \mathrm{ml}$ werden $150 \mathrm{ml}$ Petrolether zugesetzt und die unlöslichen Bestandteile abfiltriert. Einengen und anschließende Vakuumdestillation liefert Verbindung $\mathbf{1 0}$ als gelbe Flüssigkeit.

Verbindung 10: Kp. (90 mbar): $115-122^{\circ} \mathrm{C}$. Ausbeute $11.6 \mathrm{~g}(70 \%)$. ${ }^{1} \mathrm{H}-\mathrm{NMR}\left(\mathrm{CDCl}_{3}\right): \delta=-0.03(\mathrm{~s}$, 9H, $\mathrm{SiMe}_{3}$ ); 1.86 (s, 6H, C(1,4)-Me); 1.99 (s, 3H, C(2)$\mathrm{Me}) ; 2.71(\mathrm{~s}, 1 \mathrm{H}, \mathrm{C}(5)-\mathrm{H}) ; 6.07(\mathrm{~s}, 1 \mathrm{H}, \mathrm{C}(3)-\mathrm{H}) .{ }^{13} \mathrm{C}$ $\operatorname{NMR}\left(\mathrm{CDCl}_{3}\right): \delta=-1.88\left(\mathrm{SiMe}_{3}\right) ; 12.40 ; 14.23 ; 16.74$ $(\mathrm{C}(1,2,4)-\mathrm{Me}) ; 56.40 \quad(\mathrm{C}(5)) ; 130.83 \quad(\mathrm{C}(3)) ; 134.12$ $\left(\mathrm{C}(1,4) ; 141.07(\mathrm{C}(2)) .{ }^{29} \mathrm{Si}-\mathrm{NMR}\left(\mathrm{CDCl}_{3}\right): \delta=0.95\right.$ $\left(\mathrm{SiMe}_{3}\right)$. MS: $m / z(\%)=180(17.2)(M)^{+}, 165(5.6)(M$ $\left.-\mathrm{CH}_{3}\right)^{+}, 73(100)\left(\mathrm{SiMe}_{3}\right)^{+}$. Gef.: C, 71.77; H, 10.99 . $\mathrm{C}_{11} \mathrm{H}_{20} \mathrm{Si}(180.37)$ ber.: C, 73.25; H, 11.18\%.

3.11. 5,5-Bis-(trimethylsilyl)-1,2,4-trimethyl-1,3-cyclopentadien (11)

Man löst $5.32 \mathrm{~g}$ ( $29.5 \mathrm{mmol}) 10$ in $50 \mathrm{ml}$ THF, tropft bei RT $19.5 \mathrm{ml}(1.60 \mathrm{~m}$ in $\mathrm{n}$-Hexan, $31.2 \mathrm{mmol}){ }^{\mathrm{n}} \mathrm{BuLi}$ langsam hinzu und rührt die farblose Suspension $16 \mathrm{~h}$ bei RT. Nach der Zugabe von $4.15 \mathrm{~g}(38.2 \mathrm{mmol})$ $\mathrm{Me}_{3} \mathrm{SiCl}$ wird noch $3 \mathrm{~d}$ bei RT gerührt. Übliche Aufarbeitung und Destillation im Vakuum liefert 11 als farbloses hochvikoses Öl.

Verbindung 11: Kp. (1 mbar): $80-100^{\circ} \mathrm{C}$. Ausbeute 7.09 g (95\%). ${ }^{1} \mathrm{H}-\mathrm{NMR}\left(\mathrm{CDCl}_{3}\right):$ 11a: $\delta=-0.05(\mathrm{~s}$, $18 \mathrm{H}$, Si-Me); 1.83; 1.93; 2.05 (s, jeweils 3H, Cp-Me); 6.13 (s, 1H, Cp-H). 11b: $\delta=-0.03$ (s, 9H, C(5)-SiMe ${ }_{3}$; 0.20 (s, 9H, C(3)-SiMe ${ }_{3}$ ); 1.88; 1.90; 2.05 (s, jeweils $3 \mathrm{H}$, $\mathrm{Cp}-\mathrm{Me}) ; 3.08$ (s, $1 \mathrm{H}, \mathrm{C}(5)-\mathrm{H}) .{ }^{13} \mathrm{C}-\mathrm{NMR}\left(\mathrm{CDCl}_{3}\right)$ : 11a: $\delta=-0.41$ (Si-Me), 12.51; 15.09; 17.63 (Cp-Me); 57.04 (C(5)); $132.61(C(3)) ; 135.44 ; 135.72 ; 141.28(C(1,2,4))$. ${ }^{29} \mathrm{Si}-\mathrm{NMR}\left(\mathrm{CDCl}_{3}\right)$ : 11a: $\delta=-1.5(\mathrm{Me}-\mathrm{Si})$. MS: $m / z$ $(\%)=252(35.4)(M)^{+} ; 237(11.5)(M-\mathrm{Me})^{+} ; 164$ (7.2) $\left(M-\mathrm{SiMe}_{3}\right)^{+} ; 73(100)\left(\mathrm{SiMe}_{3}\right)^{+} ; 59(13.7)$ 
$\left(\mathrm{Me}_{2} \mathrm{HSi}\right)^{+}$. Gef.: C, 66.55; $\mathrm{H}, 11.10 . \mathrm{C}_{14} \mathrm{H}_{28} \mathrm{Si}_{2}(252.55)$ ber.: C, 66.58 ; H, $11.18 \%$.

3.12. 3,5-Bis-(trimethylsilyl)-5-trimethylstannyl-1,2,4-trimethyl-1,3-cyclopentadien (12)

Man löst $2.80 \mathrm{~g}(11.1 \mathrm{mmol}) 11$ in $20 \mathrm{ml} \mathrm{THF}$, tropft bei RT $7 \mathrm{ml}(1.6 \mathrm{~m}$ in $\mathrm{n}$-Hexan, $11.2 \mathrm{mmol}){ }^{\mathrm{n}} \mathrm{BuLi}$ langsam hinzu und ruihrt $16 \mathrm{~h}$ bei RT. Nach der $\mathrm{Zu}$ gabe von $2.40 \mathrm{~g}(12.0 \mathrm{mmol}) \mathrm{Me}_{3} \mathrm{SnCl}$ in $10 \mathrm{ml} \mathrm{THF}$ wird noch $3 \mathrm{~h}$ bei RT gerührt. Nach üblicher Aufarbeitung und Destillation im Vakuum erhält man 12 als farbloses hochviskoses Öl.

Verbindung 12: Ausbeute $3.89 \mathrm{~g}(84 \%)$. ${ }^{1} \mathrm{H}-\mathrm{NMR}$ $\left(\mathrm{CDCl}_{3}\right): \delta=-0.02 \quad\left(\mathrm{~s}, \quad 9 \mathrm{H}, \quad{ }^{2} J\left({ }^{119} \mathrm{Sn}-\mathrm{H}\right)=52.0\right.$, $\left.{ }^{2} J\left({ }^{117} \mathrm{Sn}-\mathrm{H}\right)=50.0 \mathrm{~Hz}, \mathrm{Sn}-\mathrm{Me}\right) ; 0.09$ (s, $\left.18 \mathrm{H}, \mathrm{Si}-\mathrm{Me}\right)$; 1.92 (s, 18H, Cp-Me); 2.09 (s, 3H, Cp-Me). ${ }^{13} \mathrm{C}-\mathrm{NMR}$ $\left(\mathrm{CDCl}_{3}\right): \delta=-7.9\left({ }^{1} J\left({ }^{117} \mathrm{Sn}-\mathrm{C}\right)=324 \mathrm{~Hz}, \mathrm{Sn}-\mathrm{Me}\right)$; 1.12 (Si-Me); 14.41; 18.7 (Cp-Me); 99.5 (C(5)); 136.12; 149.5 (C(vinyl)). ${ }^{29} \mathrm{Si}-\mathrm{NMR}\left(\mathrm{CDCl}_{3}\right): \delta=-6.5(\mathrm{Me}-\mathrm{Si})$. ${ }^{229} \mathrm{Sn}-\mathrm{NMR}\left(\mathrm{CDCl}_{3}\right): \delta=16.2(\mathrm{Me}-\mathrm{Sn})$. MS: $m / z(\%)$ $=416(7.6)(M)^{+} ; 401(7.4)(M-\mathrm{Me})^{+} ; 236(100)(M$ - $\left.\mathrm{SnMe}_{4}\right)^{+} ; 165(37.1)\left({ }^{120} \mathrm{SnMe}_{3}\right)^{+} ; 163(23.9)\left({ }^{118} \mathrm{Sn}-\right.$ $\left.\mathrm{Me}_{3}\right)^{+} ; 73(98.6)\left(\mathrm{SiMe}_{3}\right)^{+}$; $59\left(\mathrm{Me}_{2} \mathrm{HSi}\right)^{+}$. Gef.: C, 47.68; $\mathrm{H}, 8.22$. $\mathrm{C}_{16} \mathrm{H}_{36} \mathrm{Si}_{2} \mathrm{Sn}$ (415.34) ber.: $\mathrm{C}, 49.16$; $\mathrm{H}$, $8.74 \%$.

3.13. 5-Pentamethyldisilanyl-1,2,4-trimethyl-1,3-cyclopentadien (13)

Man löst $10.82 \mathrm{~g}$ (100 mmol) 1,2,4-Trimethyl-1,3cyclopentadien in $250 \mathrm{ml}$ THF, tropft bei $0^{\circ} \mathrm{C} 63 \mathrm{ml}$ $(1.60 \mathrm{~m} \text { in } \mathrm{n}-\mathrm{Hexan}, 100.8 \mathrm{mmol})^{\mathrm{n}} \mathrm{BuLi}$ langsam hinzu und rührt die farblose Suspension $16 \mathrm{~h}$ bei RT. Nach der Zugabe von $16.68 \mathrm{~g}(100.9 \mathrm{mmol}) \mathrm{Me}_{5} \mathrm{Si}_{2} \mathrm{Cl}$ wird noch $18 \mathrm{~h}$ unter Rückfluß erhitzt. Übliche Aufarbeitung und Destilation im Vakuum liefert 13 als gelbe Flüssigkeit.

Verbindung 13: Kp. (0.06 mbar): $40-41^{\circ} \mathrm{C}$. Ausbeute $19.0 \mathrm{~g}(80 \%)$. ${ }^{1} \mathrm{H}-\mathrm{NMR}\left(\mathrm{CDCl}_{3}\right): \delta=-0.04(\mathrm{~s}, 9 \mathrm{H}$, $\mathrm{SiMe}_{3}$ ); 0.23 (s, 6H, C(5)-SiMe $)_{2} ; 1.86 ; 1.92 ; 2.04$ (s, jeweils $3 \mathrm{H}, \mathrm{Me}-\mathrm{C}($ vinyl)); 2.99(b) (s, $1 \mathrm{H}, \mathrm{H}-\mathrm{C}(5)) ; 6.00$ (s, 1H, H-C(3)). ${ }^{13} \mathrm{C}-\mathrm{NMR}\left(\mathrm{CDCl}_{3}\right): \delta=-2.19 ;-1.41$ (Si-Me); 12.66; 14.53; 17.02 (Me-Cp); 55.83 (C(5)); 130.91 (C(3)); 133.8; 135.11; 141.94 (C(vinyl)). ${ }^{29}$ Si-NMR $\left(\mathrm{CDCl}_{3}\right): \delta=-18.2\left(\mathrm{SiMe}_{3}\right) ;-13.1(\mathrm{Cp}-\mathrm{Si}) . \mathrm{MS}: m / z$ $(\%)=238(26)(M)^{+} ; 165(81)\left(M-\mathrm{SiMe}_{3}\right)^{+} ; 131(77)$ $\left(\mathrm{Me}_{5} \mathrm{Si}_{2}\right)^{+} ; 73(100)\left(\mathrm{Me}_{3} \mathrm{Si}\right)^{+}$. Gef.: C, 65.56; H, 10.98. $\mathrm{C}_{13} \mathrm{H}_{26} \mathrm{Si}_{2}$ (238.52) ber.: C, 65.46; $\mathrm{H}, 10.99 \%$.

3.14. 5,5-Bis-(pentamethyldisilanyl)-1,2,4-trimetyl-1,3cyclopentadien (14)

Man löst $9.54 \mathrm{~g}$ (40 mmol) 13 in $180 \mathrm{ml}$ THF, tropft bei $0^{\circ} \mathrm{C} 25 \mathrm{ml}(1.60 \mathrm{~m}$ in $\mathrm{n}$-Hexan, $40.0 \mathrm{mmol}){ }^{\mathrm{n}} \mathrm{BuLi}$ langsam hinzu und rührt die gelbe Lösung $16 \mathrm{~h}$ bei RT. Nach der Zugabe von $6.78 \mathrm{~g}$ (40.5 mmol) $\mathrm{Me}_{5} \mathrm{Si}_{2} \mathrm{Cl}$ wird noch $16 \mathrm{~h}$ gerührt. Übliche Aufarbeitung und Destillation im Vakuum liefert 14 als gelbe Flüssigkeit.

Verbindung 14: Kp. (0.06 mbar): $90-120^{\circ} \mathrm{C}$. Ausbeute $11.7 \mathrm{~g}(73 \%)$. ${ }^{1} \mathrm{H}-\mathrm{NMR}\left(\mathrm{CDCl}_{3}\right): \delta=-0.18$ (s, $18 \mathrm{H}, \mathrm{SiMe}_{3}$ ); 0.34 (s, 12H, $\left.\mathrm{SiMe}_{2}\right) ; 1.85 ; 1.98 ; 2.12$ (s, jeweils $3 \mathrm{H}$, Me-C(vinyl)); $6.14(\mathrm{~s}, 1 \mathrm{H}, \mathrm{H}-\mathrm{C}(3))$. ${ }^{13} \mathrm{C}$ NMR $\left(\mathrm{CDCl}_{3}\right): \delta=-1.04 ;-0.27 ;-0.10(\mathrm{Si}-\mathrm{Me})$; 13.07; 15.83; 18.44 (Me-Cp); 55.22 (C(5)); 133.40 (C(3)); 135.68; $137.18 ; 142.98$ (C(vinyl)). ${ }^{29} \mathrm{Si}-\mathrm{NMR}\left(\mathrm{CDCl}_{3}\right)$ : $\delta=-17.8\left(\mathrm{SiMe}_{3}\right) ;-13.7(\mathrm{Cp}-\mathrm{Si}) . \mathrm{MS}: m / z(\%)=368$ (9.7) $(M)^{+} ; 295(38.3)\left(M-\mathrm{SiMe}_{3}\right)^{+} ; 131$ (25.9) $\left(\mathrm{Me}_{5} \mathrm{Si}_{2}\right)^{+} ; 73(100)\left(\mathrm{Me}_{3} \mathrm{Si}\right)^{+}$. Gef.: C, 56.62; H, 10.01 . $\mathrm{C}_{18} \mathrm{H}_{40} \mathrm{Si}_{4}$ (368.86) ber.: C, 58.61; $\mathrm{H}, 10.93 \%$.

\subsection{5-Pentamethyldisilanyl-1,2,3,4-tetramethyl-1,3-} cyclopentadien (15)

Man löst $9.65 \mathrm{~g}(80.0 \mathrm{mmol})$ Tetramethyl-1,3-cyclopentadien in $300 \mathrm{ml} \mathrm{THF}$, tropft bei $0^{\circ} \mathrm{C} 50 \mathrm{ml}{ }^{\mathrm{n}} \mathrm{BuLi}$ $(1.60 \mathrm{~m}$ in $\mathrm{n}$-Hexan, $80.0 \mathrm{mmol}$ ) langsam hinzu und rührt die farblose Suspension $8 \mathrm{~h}$ bei RT. Nach der Zugabe von $13.35 \mathrm{~g}(80.0 \mathrm{mmol}) \mathrm{Me}_{5} \mathrm{Si}_{2} \mathrm{Cl}$ wird $8 \mathrm{~h}$ unter Rückfluß erhitzt und anschließend $16 \mathrm{~h}$ bei RT gerührt. Übliche Aufarbeitung und Destillation im Vakuum liefert 15 als gelbe Flüssigkeit. Verbindung 15: Kp. $(0.2 \mathrm{mbar}): 64^{\circ} \mathrm{C}$. Ausbeute $13.27 \mathrm{~g}(66 \%)$. ${ }^{1} \mathrm{H}-\mathrm{NMR}\left(\mathrm{CDCl}_{3}\right): \delta=-0.06\left(\mathrm{~s}, 9 \mathrm{H}, \mathrm{SiMe}_{3}\right) ; 0.28(\mathrm{~s}$, $6 \mathrm{H}, \mathrm{C}(5)-\mathrm{SiMe}_{2}$ ); 1.86 ; 1.99 (s, jeweils $6 \mathrm{H}, \mathrm{Me}-\mathrm{C}(\mathrm{vinyl})$ ); 2.96(b) (s, $1 \mathrm{H}, \mathrm{H}-\mathrm{C}(5)) .{ }^{13} \mathrm{C}-\mathrm{NMR}\left(\mathrm{CDCl}_{3}\right): \delta=-1.73$ (Si-Me); 11.28; 14.63 (Me-Cp); 55.04 (C(5)); 133.99; 135.10 (C(vinyl)). ${ }^{29} \mathrm{Si}-\mathrm{NMR} \quad\left(\mathrm{CDCl}_{3}\right): \delta=-18.9$ $\left(\mathrm{SiMe}_{3}\right) ;-13.1$ (Cp-Si). MS: $m / z(\%)=251(41.5)(M$ $-\mathrm{H})^{+} ; 179(30.3)\left(M-\mathrm{SiMe}_{3}\right)^{+} ; 131(100)\left(\mathrm{Me}_{5} \mathrm{Si}_{2}\right)^{+}$; $63(69.2)\left(\mathrm{Me}_{3} \mathrm{Si}\right)^{+}$. Gef.: $\mathrm{C}, 66.49 ; \mathrm{H}, 10.77 . \mathrm{C}_{14} \mathrm{H}_{28} \mathrm{Si}_{2}$ (252.55) ber.: C, 66.58; II, 11.17\%.

\subsection{5,5-Bis-(pentamethyldisilanyl)-1,2,3,4-tetramethyl- 1,3-cyclopentadien (16)}

$\mathrm{Zu}$ einer Suspension von $1.09 \mathrm{~g}(8.00 \mathrm{mmol}) \mathrm{C}_{8} \mathrm{~K}$ in $30 \mathrm{ml}$ THF gibt man eine Lösung von $1.43 \mathrm{~g}(3.90$ mmol) 14 in $10 \mathrm{ml}$ THF und rührt $2 \mathrm{~h}$ bei RT. Nach der Zugabe von $1.20 \mathrm{~g}(8.2 \mathrm{mmol}) \mathrm{CH}_{3} \mathrm{I}$ wird weitere $16 \mathrm{~h}$ gerührt. Man engt im Vakuum ein, fügt $50 \mathrm{ml}$ n-Hexan hinzu, filtriert vom unlöslichen Rückstand über Kieselgel ab und engt im Vakuum ein. Kristallisation aus $\mathrm{CH}_{2} \mathrm{Cl}_{2} / \mathrm{CH}_{3} \mathrm{CN}$ liefert 16 als farblose Kristalle.

Verbindung 16: Ausbeute: $640 \mathrm{mg}$ (43\%). ${ }^{1} \mathrm{H}-\mathrm{NMR}$ $\left(\mathrm{CDCl}_{3}\right): \delta=-0.26\left(\mathrm{~s}, 18 \mathrm{H}, \mathrm{SiMe}_{3}\right) ; 0.32(\mathrm{~s}, 12 \mathrm{H}$, $\left.\mathrm{SiMe}_{2}\right) ; 1.78 ; 1.98\left(2 \mathrm{~s}\right.$, jeweils $6 \mathrm{H}$, Me-C(vinyl)). ${ }^{13} \mathrm{C}-$ NMR $\left(\mathrm{CDCl}_{3}\right): \delta=-1.52 ;-0.20 ; 1.02(\mathrm{Me}-\mathrm{Si}) ; 11.72$; 15.76 (Me-Cp); 53.6 (C(5)); 135.66; 136.59 (C(vinyl)). ${ }^{29} \mathrm{Si}-\mathrm{NMR}\left(\mathrm{CDCl}_{3}\right): \delta=-18.4\left(\mathrm{SiMe}_{3}\right) ;-14.1(\mathrm{Si}-\mathrm{Cp})$. MS: $m / z(\%)=382(15.1)(M)^{+} ; 309(100)(M-$ $\left.\mathrm{SiMe}_{3}\right)^{+} ; 131(33.9)\left(\mathrm{Me}_{5} \mathrm{Si}_{2}\right)^{+} ; 73(97.2)\left(\mathrm{SiMe}_{3}\right)^{+}$. 
5.17. 5-Pentamethyldisilanyl-1,2,3,4,5-pentamethyl-1,3cyclopentadien (17)

$\mathrm{Zu}$ einer Suspension von $1.57 \mathrm{~g}(39.3 \mathrm{mmol})$ Kaliumsand in $80 \mathrm{ml}$ Toluol gibt man $4.80 \mathrm{~g}(39.3 \mathrm{mmol})$ Pentamethylcyclopentadien und erhitzt $16 \mathrm{~h}$ unter Rückfluß. Nach der Zugabe von $6.55 \mathrm{~g}(40.5 \mathrm{mmol})$ $\mathrm{Me}_{5} \mathrm{Si}_{2} \mathrm{Cl}$ wird weitere $24 \mathrm{~h}$ unter Rückfluß erhitzt. Übliche Aufarbeitung und Destillation im Vakuum liefert 17 als gelbe Flüssigkeit.

Verbindung 17: Kp. (0.4 mbar): $60-65^{\circ} \mathrm{C}$. Ausbeute $5.25 \mathrm{~g}(50 \%)$. ${ }^{1} \mathrm{H}-\mathrm{NMR}$ (Toluol- $d_{8}$ ): $0^{\circ} \mathrm{C}: \delta=-0.08(\mathrm{~s}$, 9H, $\mathrm{SiMe}_{3}$ ); 0.14 (s, 6H, SiMe ${ }_{2}$ ); 1.14 (s, 3H, Me-C(5)); 1.78 (s, 6H, Me-C(vinyl)); 1.81 (s, 6H, Me-C(vinyl)). ${ }^{13} \mathrm{C}-\mathrm{NMR}\left(\mathrm{CDCl}_{3}\right): \delta=-3.22 ;-1.50$ (Si-Me); 11.5(b) (Me-Cp); 133.9(b) (C(vinyl)); 139.6(b) (C(vinyl). ${ }^{29} \mathrm{Si}-$ NMR $\left(\mathrm{CDCl}_{3}\right): \delta=-18.9\left(\mathrm{SiMe}_{3}\right) ;-9.2(\mathrm{C}(5)-\mathrm{Si})$. MS: $m / z(\%)=266(23.0)(M)^{+} ; 193(48.3)(M-$ $\left.\mathrm{SiMe}_{3}\right)^{+} ; 131(77.5)\left(\mathrm{Me}_{5} \mathrm{Si}_{2}\right)^{+} ; 73(100)\left(\mathrm{SiMe}_{3}\right)^{+}$. Gef.: C, 67.14; $\mathrm{H}, 11.37 . \mathrm{C}_{15} \mathrm{H}_{30} \mathrm{Si}_{2}$ (266.57) ber.: C, $67.59 ; \mathrm{H}, 11.34 \%$.

\section{Literatur und Bemerkungen}

1 (a) P.B. Hitchcock, M.F. Lappert und S. Prashar, J. Organomet. Chem., 413 (1991) 79 und dort zitierte Literatur; (b) P. Jutzi, J.
Organomet. Chem., 400 (1990) 1 und dort zitierte Literatur; (c) J. Okuda, Top. Curr. Chem., 160 (1991) 97 und dort zitierte Literatur.

2 P.G. Gassman und P.A. Deck, Organometallics, 11 (1992) 959.

3 M. Kumada, K. Mimura, M. Ishikawa und K. Shiina, Tetrahedron Lett., $I$ (1965) 83.

4 N.M. Sergejev, G.I. Avramenko, A.V. Kisin, V.A. Korenevsky und Yu.A. Ustynyuk, J. Organomet. Chem., 32 (1971) 55.

5 Yu.A. Ustynyuk, A.V. Kisin, I.M. Pribytkova, A.A. Zenkin und N.D. Antonova, J. Organomet. Chem., 42 (1972) 47.

6 Yu.A. Ustynyuk, Yu.N. Luzikov, V.I. Mistlarvsky, A.A. Azizov und I.M. Pribytkova, J. Organomet. Chem., 96 (1975) 335.

7 P. Jutzi und J. Sauer, J. Organomet. Chem., 50 (1973) C29.

8 Die Messung erfolgte auf einem Siemens P2 ${ }_{1}$ Diffraktometer (Mo K $\alpha$-Strahlung, Graphitmonochromator). Summenformel $\mathrm{C}_{25} \mathrm{H}_{62} \mathrm{Si}_{8}$, Kristallsystem tetragonal, Raumgruppe $14_{1} / a, a=$ 19.263(10), $c=21.540(15) \AA, V=8003(5) \AA^{3}, Z=8$, Dichte (ber.) $0.975 \mathrm{~g} \mathrm{~cm}^{-3}, \mu=2.74 \mathrm{~cm}^{-1}, F(000)=2592, T=293 \mathrm{~K}, 2 \theta-$ Bereich $3 \leq 2 \theta \leq 55,4592$ unabhängige Reflexe gemessen, davon 1490 beobachtet $(F>4.0 \sigma(F))$; Strukturlösung mit DirektenMethoden. Alle Nicht-Wasserstoffatome anisotrop mittels Methode der kleinsten Fehlerquadrate verfeinert (150 Parameter); Wasserstoffatome an berechneten Positionen. $R=0.0762, R_{\mathrm{w}}=$ 0.0484 (Wichtungsschema $w^{-1}=\sigma^{2}(F)$ ). Die Daten zur Kristallstrukturuntersuchung sind unter der Hinterlegungsnummer CSD57146 beim Fachinformationszentrum Karlsruhe, Gesellschaft für wissenschaftlich-technische Informationen $\mathrm{mbH}$, D-76344 Eggenstein-Leopoldshafen, hinterlegt.

9 Publikation in Vorbereitung.

10 R. Csuk, Nachr. Chem. Tech. Lab., 35 (1987) 828. 